Skjæveland, Martin G., et al. "Engineering ontology-based access to real-world data sources." Web Semantics: Science, Services and Agents on the World Wide Web 33 (2015): 112-140.

(c) 2015. This manuscript version is made available under the CC-BY-NC-ND 4.0 license http://creativecommons.org/licenses/by-nc-nd/4.0/ 


\title{
Engineering Ontology-Based Access to Real-World Data Sources
}

\author{
Martin G. Skjæveland*, Martin Giese, Dag Hovland, Espen H. Lian, Arild Waaler \\ Department of Informatics, University of Oslo, Pb. 1080 Blindern, 0316 Oslo, Norway
}

\begin{abstract}
The preparation of existing real-world datasets for publication as high-quality semantic web data is a complex task that requires the concerted execution of a variety of processing steps using a range of different tools. Faced with both changing input data and evolving requirements on the produced output, we face a significant engineering task for schema and data transformation. We argue that to achieve a robust and flexible transformation process, a high-level declarative description is needed, that can be used to drive the entire tool chain. We have implemented this idea for the deployment of ontology-based data access (OBDA) solutions, where semantically annotated views that integrate multiple data sources on different formats are created, based on an ontology and a collection of mappings. Furthermore, we exemplify our approach and show how a single declarative description helps to orchestrate a complete tool chain, beginning with the download of datasets, and through to the installation of the datasets for a variety of tool applications including data and query transformation processes and reasoning services. Our case study is based on several publicly available tabular and relational datasets concerning the operations of the petroleum industry in Norway. We include a discussion of the relative performance of the used tools on our case study, and an overview of lessons learnt for practical deployment of OBDA on real-world datasets.
\end{abstract}

Keywords: Ontology-based data access, Engineering process, Case study, Query evaluation

\section{Introduction}

Building a large software system 'from scratch' is a complex task. What used to be a matter of writing code into a text file, and then running a compiler, is now an undertaking that can involve numerous steps, including code generation, downloading specific versions of hundreds of required libraries, automated testing, and more. As this process became more complex, tools emerged to support it, like MAKE [1], ANT [2] and MAVEN [3], that are based on configuration files that abstract away from some of the details and that describe the construction process in a more declarative way. The more complicated these build processes became, the more declarative and abstract also the configuration files.

Compare this to the task of preparing information for publication as RDF [4]. This used to amount to writing some triples into a text file. For larger sets, a triple store can be used, that grants access through a SPARQL [5] endpoint, and possibly some Linked Data [6] front-end. But in the present work, we are interested in ontology-based data access (OBDA), in a sense to be made precise below, as a sophisticated method for publishing high-quality semantic datasets. Using OBDA on real-world problems, we quickly notice that also semantic technologies have departed from the state of innocence: done properly, the

\footnotetext{
${ }^{*}$ Corresponding author.

Email addresses: martige@ifi.uio.no (Martin G. Skjæveland), martingi@ifi.uio.no (Martin Giese), hovland@ifi.uio.no (Dag Hovland), elian@ifi.uio.no (Espen H. Lian), arild@ifi.uio.no (Arild Waaler)
}

process will involve a variety of steps for cleansing, transforming, and mapping data, generating (bootstrapping) ontologies for existing data schemata, improving them, managing specifications of mappings that relate ontology concepts to the data, and more. For all these different tasks, the OBDA system designers are left with an array of tools spanning from simple text editors to complex ontology editors and large-scale triple stores with automated reasoning support. Most often, this preparation of data and ontology infrastructure is not a one-shot effort: data and schemata will be updated, new data cleaning tasks become necessary, further improvements of the ontology called for, etc. One is then faced with a tangle of diverging schemata, ontologies, configuration files and scripts. The management of the whole transformation and installation process becomes a significant engineering challenge in its own right.

In this work we suggest learning from the software engineering discipline: to ensure a flexible and robust transformation process, a high-level declarative description of the transformation process is required, that can be used to drive the entire tool chain. This configuration specification is itself an engineering artefact, that concentrates in one place all the information that is needed to make the individual tools cooperate. As such, it can benefit from the full range of engineering possibilities, like explicit, localised documentation, version control, the possibility to devise tools to support the creation of the configuration, and more.

Moreover, once the declarative description is in place it provides us with a framework for prototyping and testing the relative performance of different OBDA architectures and components. Consistent with the design of the architecture one must select 
components for use from a number of available candidates. But how do we know what components fit their purpose best? The performance of these components is typically evaluated in more or less idealised "laboratory tests" with both data and queries provided by database benchmark tools such as the Lehigh University Benchmark [7] and the Berlin SPARQL Benchmark [8], ${ }^{1}$ for instance the studies [9-11]. Recently, benchmarks tailored for specific types of OBDA systems have been developed as well $[12,13]$. Although these benchmarks provide a comprehensive test bed for the evaluation of technical performance, they still lack the natural variance and imperfection usually encountered in real-world datasets. Hence, benchmarking gives limited information about how the components will serve in a real-world deployment like the one we are addressing. Also, it is difficult to know up-front how the components will perform in combination. What gives more information is to test the performance of the various components on typical user queries for the datasets at hand.

This is exactly what we did in our case study. More precisely, we developed a declarative description of the various stages in the OBDA data engineering process. We then applied that description in a proof of concept OBDA implementation to a data access scenario over real-world datasets with sample queries supplied from professional end users. In doing so we also prototyped different OBDA architectures and components and evaluated these on the sample queries. Before we go into more details we first define what we mean by OBDA and review some main points related to OBDA architectures.

\subsection{Materialised vs. Virtual OBDA}

We will in this article refer to OBDA as an approach to data access where users interact with, potentially multiple and disparate, data sources via SPARQL queries using a vocabulary specified in an OWL [14] ontology. The key reasoning tasks in an OBDA system are related to the evaluation of SPARQL queries over data exposed as RDF.

There are two main approaches to OBDA, depending on whether the RDF view is materialised or not. In both cases a transformation process is at the kernel of the system: in the materialised approach source data is transformed from the source systems upfront, while in the non-materialised, or virtual, approach, SPARQL queries posed by users are transformed into queries over the source data at query execution time.

The materialised approach is illustrated in Figure 1a. The source data is here transformed into a separate physical storage system, typically a triple store, that implements a materialised RDF view of the source data; in the figure the data transformation process is illustrated by the two arrows labelled 'data' from the source data to the RDF store. This transformation process is executed before the system is ready to answer any user query. A query processor then evaluates a user query $q$ over the RDF store rather than over the source data, possibly exploiting the ontology and interacting with the RDF store in various ways.

${ }^{1}$ For a list of relevant benchmarking tools, see http://www.w3.org/ wiki/RdfStoreBenchmarking.
Since query answering in this case requires no interaction with the systems hosting the source data, an OBDA system with a materialised RDF view is not constrained by the limitations of these systems. In particular this opens the possibility of adding information to the RDF store that is inferred from the source data and the ontology, such as explicating type information and identity relationships between individuals. Since this saturation of the RDF store can be performed up-front in a forward chaining reasoning process, the OBDA system may at run-time be able to answer powerful queries very efficiently. Moreover, the OBDA system can then also support queries that the source data system cannot easily handle. Consider, as an example, queries that require different records to be semantically identified as representing the same entity. Such queries can be processed by reasoning over an OWL ontology. However, the same type of queries are not directly supported by most RDBMS in use today as these systems are usually governed by the unique name assumption where different records are by assumption taken to represent distinct entities.

The virtual, i.e., non-materialised, approach to OBDA is illustrated in Figure 1b. Since the RDF view of source is virtual, there is no duplication of the source data. In this case the query processor makes use of the ontology to transform a user query $q$ into a query $q_{O}$. The latter query is simpler than $q$ in the sense that it can be interpreted without reference to implicit information in the ontology. The query $q_{O}$ results from a reasoning process where inferences from the ontology are computed at query execution time by means of query rewriting, a backwards-chaining reasoning technique. In the next stage of the transformation process, a transformer uses information encoded in so-called mappings that relate the source data to its RDF exposure. This stage unfolds $q_{O}$ into a query $q_{\mathrm{DB}}$ that makes no reference to any concepts of the ontology, and that can be executed by the source data system.

When deciding on the architecture for an OBDA system, the following points are particularly relevant:

- Is source data frequently updated? If so, the cost of keeping a materialised view synchronised with the source data should be considered. A virtual approach has significant advantages.

- Are powerful data transformations needed for the RDF view? Then a materialised approach is needed.

- Is support needed for fragments of OWL for which query rewriting is not a complete deduction method? A materialised approach may support an ontology language with sufficient expressivity.

- Is the response time for query execution critical? Then one should prototype different architectures upfront and evaluate them on typical user queries as the results are in many cases hard to predict.

In cases where some of the source data systems do not offer any query interface, a typical case in point would be CSV files, a direct virtual approach is not an option. In cases where a full transformation into RDF requires several transformation steps, 


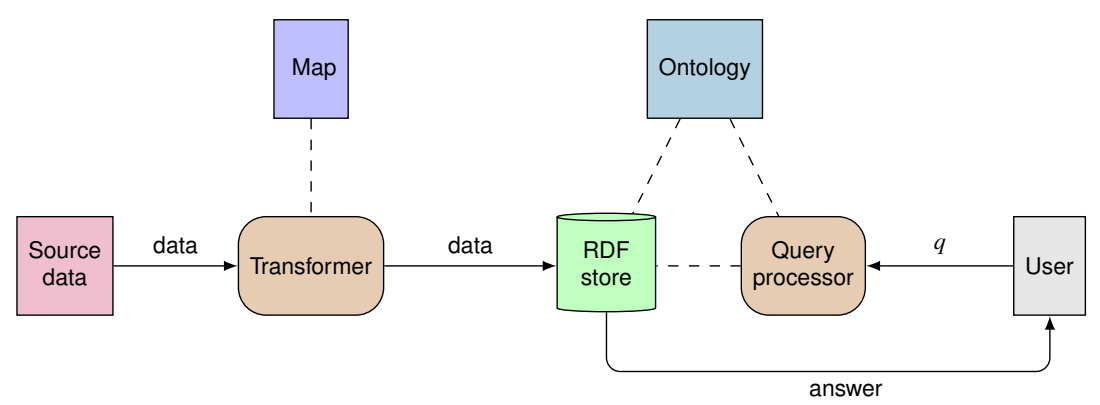

(a) The basic architecture of an OBDA implementing a materialised RDF view.

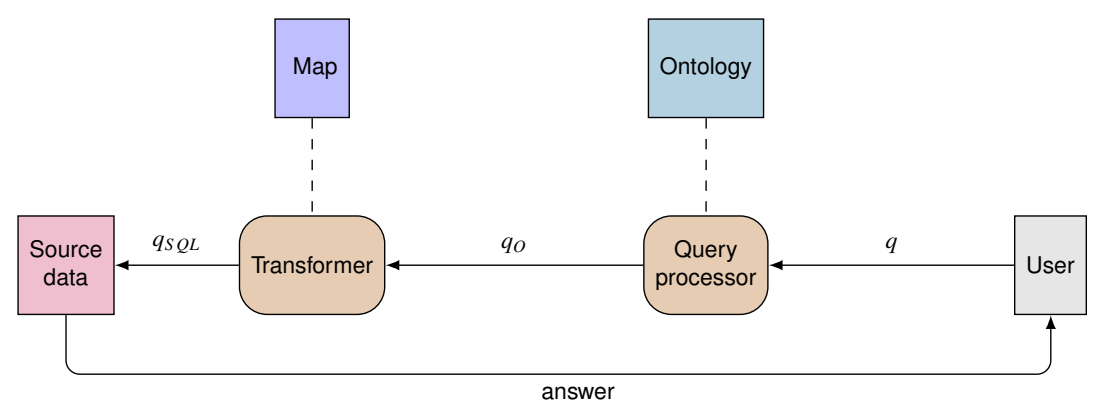

(b) The basic architecture of an OBDA system implementing a virtual RDF view.

Figure 1: Basic OBDA system architectures

one can gain some of the advantages of a virtual approach by just a lightweight transformation of the data into a system that supports query answering and then treating this system as the source.

\subsection{OBDA components}

Over the last decade, the design of appropriate ontology languages and the complexity of their associated reasoning problems have been extensively addressed [15-18]. Several components that can be used in the implementation of OBDA systems are by now also available. However, the engineering aspects of designing OBDA systems have not been given corresponding attention, and there is yet little experience derived from practical deployments of OBDA components to support access to real-world datasets in daily use. A major motivation for our work is to contribute to fill this gap.

In the following we review various architectures of OBDA systems, focusing on different choices for the transformation and reasoning processes. Relevant OBDA tools to play a part of a complete OBDA system are triple stores (virtual and physical), reasoners, and bootstrappers, and tools that transform data into RDF, often called RDFizers. ${ }^{2}$ Some tools can fill more than one category.

Virtual triple stores tools are D2RQ [19-21], Ultrawrap [10], XLWrap [22], SPARQL2XQuery [23], Spyder [24], Mastro [25], and Ontop [26]. These are OBDA tools that leave the data where it is, and transform the queries into the language of the present

\footnotetext{
${ }^{2} \mathrm{~A}$ list of RDFizers can be found at http://www.w3.org/wiki/ ConverterToRdf.
}

storage engine, e.g., SQL or XQuery. D2RQ and XLWrap do not support reasoning on the ontological level, while the others support different levels of ontological reasoning. These tools can also be used to export source data as RDF store, though that is not their primary usage. For research into virtual triple stores over relational databases, see for instance [27-30]. Physical triple stores are plentiful, we mention only two, Fuseki [31] and Stardog [32], of which only Stardog supports ontological reasoning natively. ${ }^{3}$

Ontology bootstrapping is a technique that uses a relational schema to create an ontology with classes, data and object properties, domain and range axioms, and other axioms. Populating the ontology with data values may also be part of the process. The idea of creating RDF views of relational databases was already described by the designers of the semantic web, see Berners-Lee's design note from 1998 [33]. This was the basis for dbview . py [34]. The same year, a description of mapping relational data automatically into an ontology was done by Stojanovic et al. [35]. Sequeda et al. [36] give a more recent overview of the research in this area. Implicit in these tools is some mapping between the relational data and the ontology, in newer tools these mappings are represented in the R2RML [37] format. We mention the bootstrapper RDBToOnto [38] and also the database engine Virtuoso [39], which includes bootstrapping features.

\footnotetext{
${ }^{3}$ See http://www .w3.org/wiki/LargeTripleStores for a list of triple stores that are known to tackle large datasets.
} 


\subsection{Case study}

Our case study, addressed in Section 3, targets data sources in daily use in the oil \& gas industry and can serve as a motivation for the contributions in this article. The case study contains a number of practical challenges; moreover, these challenges are of a kind that the designer of a practical OBDA system will in many cases have to cope with:

- Source data in a variety of formats. In our case, we had to cope with just under $100 \mathrm{CSV}$ files, some with a rich schema and little data, others with a small schema and much data, in addition to one poorly structured relational database.

- Source data in systems which do not offer a query interface. In our case, the CSV files were in this category.

- Frequently updated source data. In our case most of the CSV files were generated from a source that is updated on a daily basis. Even the schema of the CSV files is updated from time to time, sometimes at frequencies of less than a month.

- No available ontology covering the domain. In our case we could use two relevant domain ontologies (for stratigraphy and for geometry); otherwise we had to generate ontologies from schemata and integrate them with the domain ontologies guided by a well-established upper ontology [40]. This resulted in an ontology with approximately 400 classes and 700 roles.

- No available mappings between sources and the ontology. This is the usual situation; in our case study we generated approximately 1700 mappings.

We prototyped systems with both a materialised and a virtual architecture, where the latter dumped the CSV files into a relational database using a simple transformation. We then selected 42 typical queries for evaluation; 21 of these were supplied by working domain experts reflecting information they need in their daily work. To make this study easy to adapt and extend, we decided to test only freely available software components. As OBDA tools we used Ontop [26], Fuseki [31], D2RQ [21] and Stardog [32], and MySQL [41] was used as the underlying relational database engine.

With our specific combination of test data, test queries, and choice of tools, it turned out that:

- The virtual approach could handle 5 out of the 21 expert queries, while the materialised approach with reasoning support could handle 20 out of 21 , and all queries without reasoning enabled.

- The virtual approach could handle 12 out of the 21 technical queries, while the materialised approach with reasoning enabled could handle 18 out of 21 , but only 14 returned results when reasoning was not enabled.
For several of the queries, the virtual approach suffered from the fact that parts of the SPARQL language had not yet been implemented in Ontop at the time of evaluation. ${ }^{4}$ Another interesting observation is that for the technical queries, enabling different profiles of OWL 2 in 5 of the cases returned different result sets. This calls for a careful analysis of what OWL profile is the best choice for the case at hand.

Given the frequent update of source data in this particular case study, a virtual approach is far better from the point of view of synchronisation. This holds also in cases where the source data is CSV files, due to the fact that loading CSV files into a relational database is a much cheaper operation than a full RDF materialisation. On the other hand, we learn from the evaluation that the materialised approach can handle more of the queries. To implement an operative OBDA system based on the evaluation results of the prototypes, a designer must weigh the cost of keeping a materialised RDF view synchronised with the source data against the lack of support for some of the queries in the virtual approach.

These insights would have been impossible to know up-front. Moreover, changes in the datasets, schemata, ontologies, maturity of the used tools, etc., are likely to change the outcome. This only strengthens our point that a declarative specification of the data engineering process is required to guarantee its ability to be reproduced and adapted.

\subsection{The structure of this article}

In Section 2, we introduce our proposed language for the declarative specification of data engineering for OBDA installations, and describe the process of generating all required components from such a specification. Section 3 describes the use of this infrastructure on real-world datasets, and the challenges this implies, concluding with an empirical evaluation of the generated OBDA installations. We evaluate the usefulness of the OBDA systems with a series of SPARQL queries. We argue that this is the natural way of doing such an evaluation, as the query interface is the single point of interaction with the system that the user has. Section 4 concludes the paper. We include two appendices: Appendix A contains full details of the specification language and its interpretation, while Appendix B lists the queries used in the evaluation.

\section{A Declarative Description for Engineering Ontology- based Data Access}

The concrete data engineering challenge we address stems from the desire to compare the applicability of the different OBDA approaches shown in Figure 1 on a collection of raw data that is not yet prepared for semantic publishing. The datasets are described in detail in Section 3; for now it suffices to say that parts of the data reside in a static relational database, while other parts are in CSV format downloaded via HTTP. Also, parts of the datasets are regularly updated, both the schema structure and

\footnotetext{
${ }^{4}$ According to the developers of Ontop, implementing these features is future work.
} 
the data contents. For our comparison, the datasets need to be prepared for both of the architectures in Figure 1. In the case of Figure 1a, this means transforming the datasets into RDF and loading them into a triple store. In the case of Figure $1 \mathrm{~b}$, the datasets that are not readily available via a query interface, i.e., the CSV files, need to be converted and loaded into a relational database. In both cases, an ontology and mappings have to be produced that are suitable for both architectures, preferably leveraging the existing information in the data schemata.

Repeatedly performing all the necessary transformation steps manually is clearly far too laborious and error prone. But also a custom-built automated process, based on ad-hoc scripts where the transformations are programmatically specified is error prone, and scales badly. We therefore quickly identified the need for a central generic specification that can be used to drive the construction of the different OBDA system components for all data sources. With a single declarative specification that describes how to create a relational database, an ontology and mappings from the source data, it is easy to develop and customise the different individual components in a consistent and holistic manner, while keeping all parts of the combined OBDA system synchronised. The outputs of the transformations are produced by software that can be applied to any OBDA engineering specification. Supporting new output formats required by specific tools or modifying existing output produced by the specification is simple and transparent as this can be controlled by small independent software programs. This fosters an environment that is suitable for rapid prototyping and development of complete OBDA systems.

This section describes in detail what information is encoded in the generic specification, and how the various required OBDA system components are generated from it.

\subsection{Overview of the OBDA Engineering Specification}

The OBDA engineering specification consists of:

- a language for describing the source data and the resulting OBDA components, and

- a set of rules that translate the specification and the source data into the specified output.

We have implemented the rules and orchestrated them to an automated transformation process for the specification that covers our requirements for the comparison of OBDA tools. In its current form, the specification language and implementation support source data in tabular files or relational databases. The specification gives a generic description of the data sources and details how the sources are to be translated and published as semantic web data. By developing simple tools that implement the specification and take the source data and an accompanying specification instance as input we are able to produce:

- SQL scripts that create the structure and contents of tabular data files as a relational database,

- an ontology that reflects the semantics of the database and the source data, and
- mappings between the relational database and the ontology.

Figure 2 on the following page presents an overview of the required transformations between the three representation formats of the data, i.e., from the original tabular files to a relational database, and the mappings from the database to an ontology. The vertical arrows in Figure 2 illustrate the lifting steps from tabular files to relational databases to semantic data. The horizontal arrows are part of the data model of the respective representation format. The labels at the top situate the concepts in the specification language. These labels refer to specification maps, which are explained in the section below. We explain the figure by first giving a high-level overview of the meta-model for each of the data representation formats, and how the constructs of the three representation formats are related via the lifting steps.

As to the lifting from tabular files to databases, each tabular file is assumed to contain one or more fields. ${ }^{5}$ These are mapped to a relational database as follows:

- Each file is mapped one-to-one to a database table.

- Each field is mapped one-to-one to a database column.

We assume that a database table contains one or more columns, and that a table has exactly one primary key, given by a list of one or more column names. A foreign key interacts with exactly two database columns. These constructs are lifted into ontologies as follows:

- A database table is mapped to zero or more classes.

- A column is mapped to zero or more properties and restriction axioms.

- A foreign key is mapped to zero or more properties and restriction axioms.

- A primary key is optionally mapped to one ontology key.

An ontology consists of a set of classes, properties, individuals, and axioms. An ontology key is key for one class, and is specified with a list of one or more properties. A restriction axiom specifies the use of one property in relation to one or more classes. A subclass axiom relates two classes.

Figure 2 also indicates what parts of a lifted format may be produced in a lifting step and how complete the specification of the lifting can be. The figure shows the degree of freedom that is possible (or necessary) in each transformation step to produce output of high quality, and thus how much can be automated. For the lifting of tabular files to a relational database, tables and columns are completely specified by one-to-one relationships and is therefore possible to fully automate. However, primary and foreign database keys are not possible to lift from the tabular files since this information is not represented in the tabular files, as is indicated in the figure by the lack of inwards arrows to the primary and foreign key nodes. Database keys must hence be

\footnotetext{
${ }^{5}$ We use the word 'field' to address the "columns" in a tabular file, and reserve the word 'column' for columns in a relational database table.
} 


\begin{tabular}{|c|c|c|c|}
\hline & & pecification map & \\
\hline entifier map & Entity map & Attribute map & Relation map \\
\hline
\end{tabular}

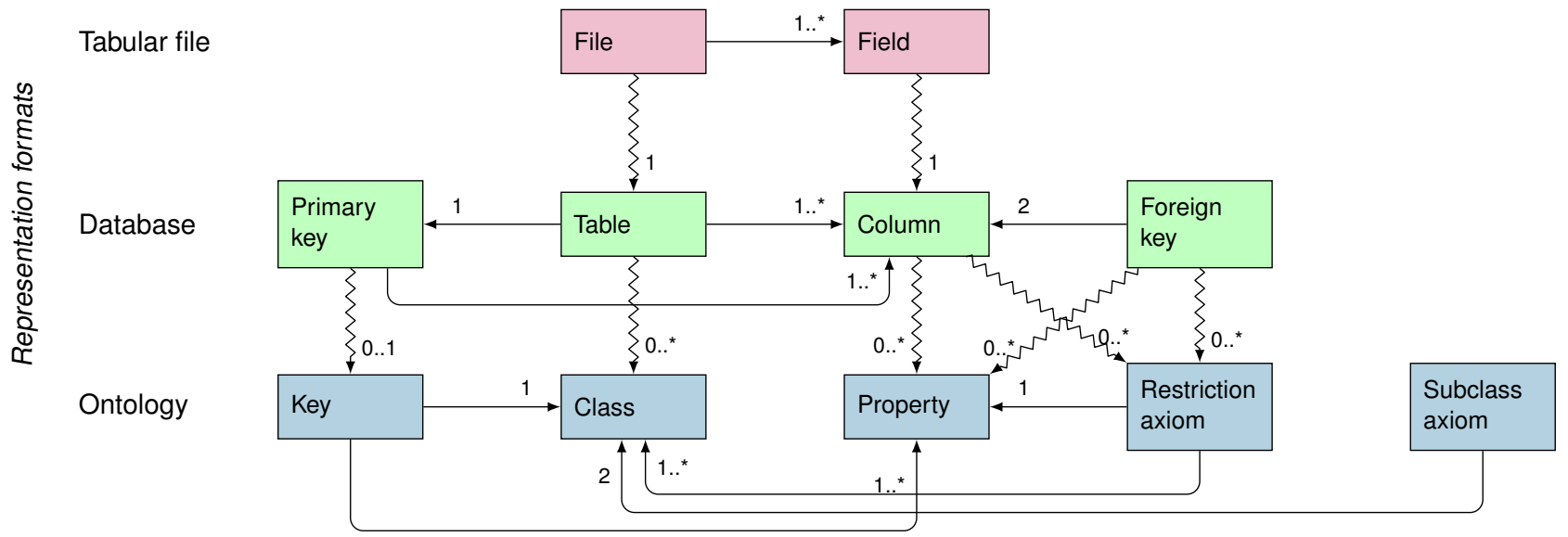

Figure 2: Overview of the transformation model of the OBDA engineering specification showing how the constructs of the three representation formats are related. The main constructs of each representation format are placed on the same level on the horizontal axis. The placement on the horizontal axis indicates which part of the specification, i.e., which specification map, is responsible for controlling the transformation. Vertical arrows indicate transformations or lifting steps between constructs of different representation formats. Horizontal arrows indicate a reference between constructs within the same format.

explicitly set in the specification. The lifting step to produce an ontology allows for considerable customisation, as seen by the cardinalities on the lifting relations between the database and the ontology; no database construct is required to be mapped to the ontology, and most database constructs may result in multiple ontology constructs. Subclass axioms are not represented in the database and must be specified especially for the ontology construction step. The degree of possible customisation in this step reflects the fact that constructing semantic models from relational models is an effort that in many cases requires significant ontological engineering.

We have constructed scripts that can bootstrap an OBDA engineering specification based on the available schema data from the sources and according to the lifting relationships in Figure 2. For the lifting step of tabular data to the relational model, the bootstrapper generates database table and column names, leaving it to the user to specify keys and further details of the table and column definition. For the step of specifying an ontology and mappings, the bootstrapper will produce a lifting based on the direct mapping specification [42], as to give the user a starting-point for further customisation.

The direct mapping specification is a precise description of how to construct an ontology from a database. It prescribes roughly that

- each table is mapped to a class,

- each table primary key is used to set the URI pattern of the instances of the corresponding class,

- each foreign key is mapped to an object property,

- each column is mapped to a data property,
- the range of the property is set according to the datatype of the database column,

- the class and property URIs are built from the respective table and column names.

In total, this makes a very crude mapping, in essence creating an ontology representation of the data model of the database, while our intention is to construct an ontology that reflects the semantics of the database contents. Some of the problems of using direct mapping in this respect are given in Figure 3, which will be explained after the constructs of the OBDA engineering specification are given. A remedy to these problems could of course be to custom-build the ontology and allow mappings expressed as arbitrary queries over the source data. The latter is possible in R2RML mapping language [37], and also other proprietary mapping languages. However, ontology development is on its own a difficult and expensive task. Now add the task of constructing and maintaining mappings that fit the ontology and sources-for sources that evolve, and the problem quickly becomes intractable. This is partly because of the sheer size of the required mappings for complex databases and hence their corresponding ontology, but also because tool support for mapping management is very scarce. Fortunately, some of the problems that are related to direct mapping can be fixed with relatively simple means. To overcome these problems, our specification language supports a variety of customisation possibilities based on simple modelling patterns that extend the direct mapping approach and that are declared in a compact and declarative manner. 


\section{Problems with direct mappings:}

1. The names used in relational databases are often not fit for an ontology. Either the names are computer-generated and unintelligible, or, in many cases, they are in plural form and unsuited for direct adaptation.

2. The structure of a database rarely represents the optimal structure of an ontology. Tables are usually organised according to normal forms to avoid duplication and update anomalies, and not to best present the semantics of the data.

3. Non-key columns in many cases represent entities and not data values, and should hence be translated to object properties and not datatype properties.

4. Some columns represent information that is best translated to type- or subclass axioms.

5. Designing URIs for individuals is not possible.

6. Reusing existing ontologies and vocabularies is not possible.

\section{Important features of the OBDA engineering specification:}

1. A URI must be specified for every map of classes and properties. A label must be set for all maps that declare new classes and properties.

2. It is possible to map a table to multiple classes, and select which columns are to be mapped for each class mappings. This is done by creating multiple entity maps per table that specify what attributes to map for the given entity.

3. It is possible to specify the property type for an attribute mapping, constructing object properties specifically, but also datatype properties and annotation properties.

4. It is possible to create subclasses based on values in the data, using subtype maps.

5. URI patterns are specified for all ontology individuals. These are used when building mappings between the database and the ontology.

6. Reusing existing ontologies are possible by setting the URI of the class or property. For each attribute map, it is possible to specify a transformation of data values into arbitrary RDF entities, including URIs.

Figure 3: Modelling features of the OBDA engineering specification, contrasted to problems that can occur from using direct mapping as the only method for ontology development from databases.

\subsection{The Specification Language}

The main concepts of the specification language reflect the key modelling constructs used in both the tabular/relational and the RDF/ontological views of the data. This corresponds to the constructs, at the schema level, needed to represent data models generated at the respective lifting steps. There are five types of specifications, these are also seen in the horizontal axis of Figure 2:

1. Entity maps relate tabular files, relational tables and ontology classes. They specify how a tabular file is mapped to a relational table, and furthermore how the table is mapped to ontology classes.

2. Identifier maps specify database primary keys and URI patterns for ontology individuals, and relate the primary keys to their corresponding ontology keys.

3. Attribute maps relate tabular file fields, relational columns and ontology properties. They specify how a field in a tabular file is mapped to database column, and additionally how the column is mapped to an ontology property and axioms.

4. Relation maps specify foreign keys and relate these to their corresponding object properties and axioms.
5. Subtype maps specify subclass information in the ontology which is generated from values in the source data.

Figure 4 details what information the specification contains for each of the five specification types. Figure 5 gives some excerpts from the specification used in our case study. As an example, consider the Entity map and line 1 of Figure 5a, which contains an entity map instance. Tabular files are by default mapped to tables with the same name, excluding the file extension. This map thus specifies that the tabular file field_operator_hst.csv shall be mapped to the table with the same name as the file, field_operator_hst, and, furthermore, that the table is mapped to the OWL class npdv:FieldOperator. The human readable label for the table and class will be "Field: Operators", and four attributes, dateSyncNPD, fldOperatorDateUpdated, fldOperatorFrom, fldOperatorTo, are selected for mapping over to the ontology description of this class. Furthermore, mappings that describe how to construct ontology individuals from the database contents may be generated. The details of these translations will be given later in this section.

The design of the language has grown out of the need for an efficient and clear representation of multiple formats. The specification language allows for representing relatively complex modelling and mapping structures from simple and compact specifications. Moreover, the structure of the language ensures that similar constructs are grouped while others are 


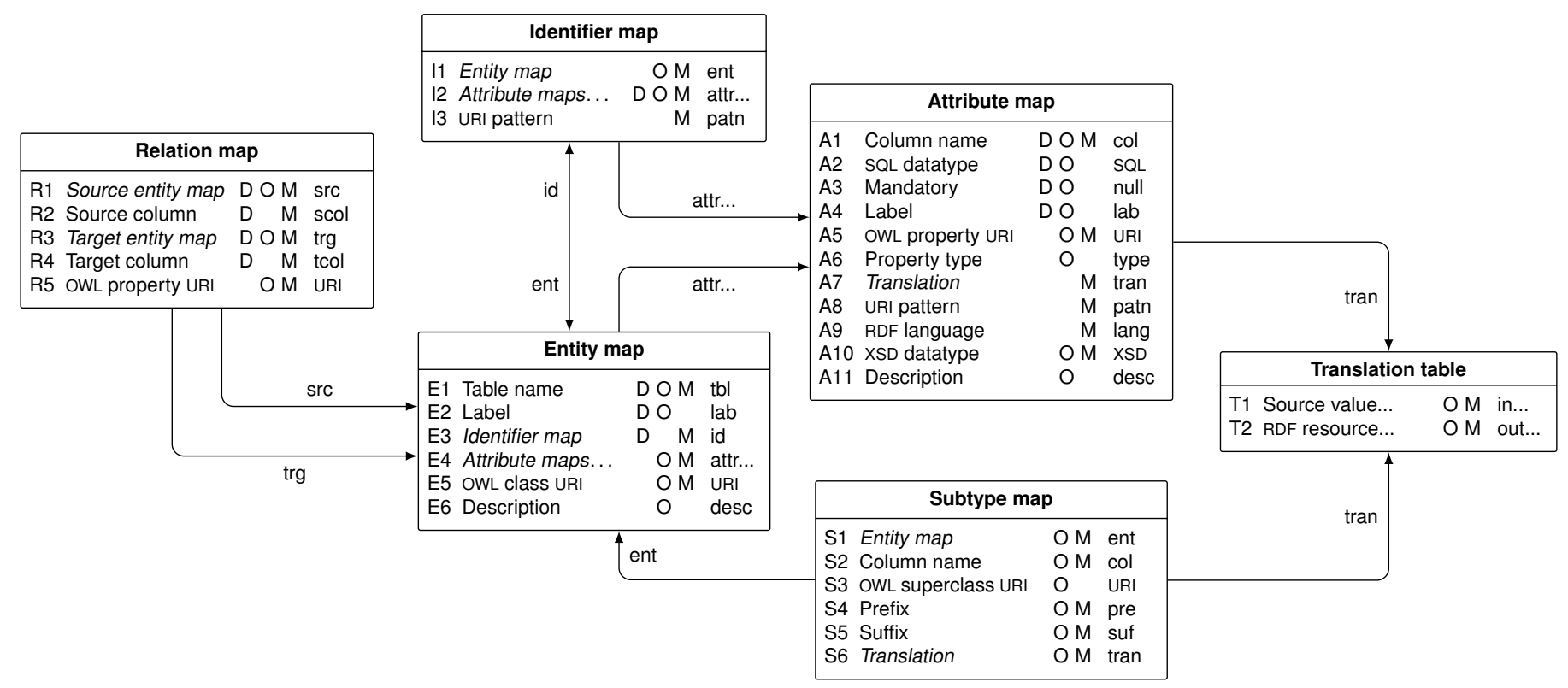

Figure 4: Schematic overview of the OBDA engineering specification, showing the five specification map types and the fields they comprise. Each field is uniquely identified with an alphanumeric label, e.g., E1, and a name, which is unique for the map type where the field occurs. An ellipsis (...) denotes that the field may contain multiple values. Fields written in italic indicate that the field is a reference to different map of the specification; this is also shown with an arrow connecting the two maps. A field is marked with $\mathrm{D}, \mathrm{O}$ or $\mathrm{M}$ if it is used in the construction of respectively database schema, ontology, or mappings. The right-most names given for each field is its shortname. This name is unique for a map and is sometimes used to improve the readability when referring to the field. Example instances of parts of the specification are found in Figure 5 .

clearly separated in order to reduce update anomaly problems of the specification, which again increases the usability of the specification language. Figure 3 lists the most important modelling features of the engineering specification and relates them to the problems of using the direct mapping specification as a single ontology construction method. Of these we highlight the ability to assign URIs for all classes, properties and individuals, and the detailed control of how database tables and columns are mapped to classes and properties of different types as absolutely necessary for producing a semantic model of reasonable quality from the relational schema. Of the more advanced features the specification allows for transforming information that not directly supported by the relational model, like specialisation and equality relationships, but which is represented in designated columns, as appropriate axioms in the ontology and mappings.

How the specification works is explained in detail in the remainder of this section, where we in turn present the three main outputs that are defined from the specification: the declaration of a relational database, an ontology and mappings between the database and ontology. A running example taken from our case study will illustrate the different outputs of the process. For the sake of readability, this section will give an informal description of the transformation process, referring to Appendix A for the formal definition. Appendix A.1 gives the syntactic requirements for a valid specification, and Appendix A.2 contains rules for translating a specification into, respectively, a relational database, an ontology and mappings.

\subsection{Representing Tabular Data Files as a Relational Database}

The process of transforming a set of tabular files into a relational database is devised so that the tabular files are faithfully represented in the database, mapping tabular files and their fields one-to-one to relational tables and columns. This is designed on purpose for the use case study in order to make the relational database an exact as possible representation of the tabular source data. However, it is of course possible to exploit this step to introduce more sophisticated transformations of the data.

The specification maps that are used in the transformation step from tabular to relational data are entity maps, identifier maps, attribute maps and relation maps. An entity map specifies a table name (E1), a label describing the table (E2) and a reference to one identifier map (E3). ${ }^{6}$ An identifier map specifies a primary key, and refers to a list of attribute maps (I2). An attribute map specifies a column, and contains a column name (A1), an SQL datatype for the column, a Boolean value indicating if a value for the column is optional or not (A3), and a label describing the column (A4). A relation map specifies a foreign key, and does this with a source column (in a table) $(\mathrm{R} 1+\mathrm{R} 2)$ and a target column $(\mathrm{R} 3+\mathrm{R} 4)$. Filenames of tabular files, without their file extension, are used as names for the corresponding tables, and similarly, field names are used as column names. Additionally, all field names in a tabular file are assumed to refer to attribute maps, hence indirectly specifying a list of attribute maps of the entity map that the tabular file is specified by.

\footnotetext{
${ }^{6}$ The parenthesis refer to the fields listed in Figure 4 on the current page. See the figure's caption for information.
} 


\begin{tabular}{|c|c|c|c|c|}
\hline Table name & OWL class URI & Label & Description & Attribute maps... \\
\hline 1 field_operator_hst & npdv:FieldOperator & "Field: Operators" & & $\begin{array}{l}\text { dateSyncNPD, } \\
\text { fldOperatorDateUpdated, } \\
\text { fldOperatorFrom, } \\
\text { fldOperatorTo }\end{array}$ \\
\hline 2 field & npdv:Field & "Field" & $\begin{array}{l}\text { "Fields on } \\
\text { the NCS" }\end{array}$ & $\begin{array}{l}\text { dateSyncNPD, } \\
\text { fldCurrentActivitySatus, } \\
\text { fldName, } \\
\text { fldNpdidField, } \\
\ldots\end{array}$ \\
\hline 3 company & $\mathrm{npdv}$ : Company & "Company" & $\begin{array}{l}\text { "Companies } \\
\text { on the NCS" }\end{array}$ & $\begin{array}{l}\text { cmpOrgNumberBrReg, } \\
\ldots\end{array}$ \\
\hline
\end{tabular}

(a) Entity map

\begin{tabular}{|c|c|c|}
\hline Entity map & URI pattern & Attribute maps... \\
\hline 1 field_operator_hst & npd:field/ $\{\$ 1\} /$ operator $/\{\$ 2\} /$ history $/\{\$ 3\} /\{\$ 4\}$ & $\begin{array}{l}\text { fldNpdidField, } \\
\text { cmpNpdidCompany } \\
\text { fldOperatorFrom } \\
\text { fldOperatorTo }\end{array}$ \\
\hline 2 field & npd:field/ $\{\$ 1\}$ & fldNpdidField \\
\hline 3 company & npd: company $/\{\$ 1\}$ & cmpNpdidCompany \\
\hline
\end{tabular}

(b) Identifier map

\begin{tabular}{|c|c|c|c|c|c|c|}
\hline & Column name & SQL datatype & OWL property URI & type & null & URI pattern \\
\hline 1 & fldName & $\operatorname{VARCHAR}(40)$ & npdv: name & $\mathrm{D}$ & 1 & \\
\hline 2 & cmpLongName & $\operatorname{VARCHAR}(200)$ & npdv: name & $\mathrm{D}$ & 1 & \\
\hline 3 & fldNpdidField & INTEGER & npdv:idNPD & $\mathrm{D}$ & 1 & \\
\hline 4 & fldOperatorFrom & DATETIME & npdv:dateFieldOperatorFrom & $\mathrm{D}$ & 1 & \\
\hline 5 & fldOperatorTo & DATETIME & npdv:dateFieldOperatorTo & $\mathrm{D}$ & 0 & \\
\hline 6 & fldNpdidField & INTEGER & & & & \\
\hline 7 & cmpNpdidCompany & INTEGER & & & & \\
\hline 8 & fldOperatorDateUpdated & DATETIME & npdv:dateUpdated & D & 0 & \\
\hline 9 & dateSyncNPD & DATETIME & npdv: dateSyncNPD & D & 1 & \\
\hline 10 & fldCurrentActivitySatus & $\operatorname{VARCHAR}(40)$ & npdv:status & $\mathrm{D}$ & 0 & \\
\hline 11 & fldOwnerKind & VARCHAR (40) & npdv:fldOwnerType & D & 1 & \\
\hline 12 & fldFactPageUrl & $\operatorname{VARCHAR}(200)$ & npdv: factPageURL & 0 & 1 & \\
\hline 13 & cmpOrgNumberBrReg & $\operatorname{VARCHAR}(100)$ & owl: sameAs & $\mathrm{OP}$ & 1 & $\begin{array}{l}\text { https://ws.brreg.no } \\
\text { /lod/data/ }\{\$ 1\}\end{array}$ \\
\hline
\end{tabular}

(c) Attribute maps. In the column 'type', D, O and OP indicate a datatype property, object property and object property pattern, respectively. In the column 'null', 1 is 'true' and 0 is 'false'.

\begin{tabular}{lllll} 
Source entity map & Source column & Target entity map & Target column & OWL property URI \\
\hline 1 field_operator_hst & cmpNpdidCompany & company & cmpNpdidCompany & npdv:fieldOperator \\
2 field_operator_hst & fldNpdidField & field & fldNpdidField & npdv:operatorForField
\end{tabular}

(d) Relation maps

\begin{tabular}{llllll} 
Entity map & Column name & OWL superclass URI & Prefix & Suffix & Translation \\
\hline 1 facility_fixed & fclKind & npdv:Facility & npdv: & Facility &
\end{tabular}

(e) Subtype maps

Figure 5: An example of an OBDA engineering specification, using a simplified excerpt of the specification developed for the use case described in Section 3. For the sake of brevity, only a sample of the available specification map fields are shown here. Each map instance is numbered for easy identification and reference in examples; this is not part of the specification. 
This information is now straight-forwardly used to specify the transformation of a set of tabular data files into a database schema as follows:

- Each entity map results in a table, with the specified table name and the label set as comment on the table. The identifier map specifies the primary key of the table, and the attribute maps specify the columns of the table.

- A primary key is constructed from an identifier map by taking all the columns of the attribute maps that the identifier map refers to as primary key columns.

- Columns are built from the attribute maps that an entity map refers to by setting the column name and SQL datatype as indicated by the map, adding the label as a comment to the column, and declaring the column as NULL or NOT NULL according to whether or not the value for the column is set as optional or not.

- Each relation map results in a foreign key that requires that values in the specified source column references values in the specified target column.

The formal description of how a database schema is constructed from the specification and the tabular source data files are given by the two database rules in Appendix subsection A.2. Database Rule 1 produces tables with columns and primary key, while Database Rule 2 constructs foreign keys.

Consider Figure 6 which contains a sample of the CSV file field_operator_hst.csv with all eight tabular field names in first row, and data in the consecutive rows, here only showing one row concerning the field operator history of the Troll field. ${ }^{7}$ The structure and metadata of the tabular file is represented in the specification, as is partly illustrated in Figure 5. The resulting database table definition of table field_operator_hst is given in Figure 7 on the following page, using syntax accepted by a MySQL database. Note that the table is defined with the same columns as the fields found in the tabular file in Figure 6 (see lines 2-2 in Figure 7), the label set in the entity map is added as comment to the table (line 2 ) and that the four columns set by the identifier map in Figure 5b constitute the primary key (line 2). Moreover, the datatypes, NOT NULL flags and comments for the columns are correctly set according to the attribute maps in Figure 5c, and the relation maps in Figure $5 \mathrm{~d}$ amount to foreign keys, as seen in lines 2 and 2 .

This database table can be loaded with the data in the tabular file, from which the structure of the table is defined, by using the SQL script in Figure 8. This script is built from the specification and uses MysQL database syntax.

\subsection{Specifying an Ontology from a Relational Database}

Different from the specification of the transformation of tabular files to a relational database, where the purpose is to let the database identically reflect the set of tabular files, the lifting

\footnotetext{
${ }^{7}$ See, e.g.,

https://en.wikipedia.org/wiki/Troll_gas_field
}

of the database to an ontology must allow for far greater customisation, as the main purpose of this step is to increase the semantic value of the data and to create a vocabulary for the OBDA interface suitable for its intended users. This includes, on the simple end, giving comprehensible names for the resulting classes and properties, and converting database constraints to ontology axioms, but also exploiting the expressiveness of the ontology language to represent the implicit semantics of the database explicitly in the ontology.

All specification maps are used for this lifting step.

- Entity maps are used to map tables to classes. They contain a class URI (E5), a list of references to attribute maps that will be associated with the class (E4), a label (E2) and a textual description or definition of the class (E6). Additionally, in order to trace the source for the class mapping, the entity map will also make use of the database table name (E1).

- Identifier maps specify ontology keys. They contain a reference to a entity map (I1) and a list of references to attribute maps (I2) which are used to select the properties will identity the given class.

- Attribute maps transform database columns to properties and restriction axioms. The fields that are used for this purpose are those that specify a URI (A5), the property type (A6), the RDF datatype (A10), if a column value is optional or not (A3), a label (A4), a textual description or definition (A11), and data about the originating database column (A1, A2). The type of the property may be set to an object, datatype or annotation property, and only for certain choices of the type will the other fields be used, for instance, an RDF datatype is only relevant if the type is a datatype property.

- Relation maps result in object properties and restriction axioms. They contain a URI (R5) and references to source and target entities (R1, R3).

- Subtype maps are used to transform the values in database columns that contain type information and specialisations into subclass axioms. The map contains fields that specify a URI (S3), a reference to an entity map (S1), a column name of the table that corresponds to the referred entity map (S2), and a possible prefix and suffix used to produce new URIs (S4, S5). A subtype map may also refer to a translation table (S6).

- A translation table specifies a one-to-one mapping between values in the database (T1) and values that will, in this transformation step, occur in the ontology (T2). A translation table is used for gaining detailed control of how database values are translated to (parts of) URIs.

We will now explain how the specification maps are used to transform a relational database into an ontology. In the following, ontology axioms are serialised using the OWL 2 Functional-Style Syntax [14]. 
1 fldName, cmpLongName, fldOperatorFrom, fldOperatorTo, fldNpdidField, cmpNpdidCompany, fldOperatorDateUpdated, dateSyncNPD

2 TROLL, A/S Norske Shell, 1986-12-15, 1996-06-19, 46437, 562, 9999-12-31, 2013-05-08

Figure 6: Excerpt of field_operator_hst.csv: Troll operator history data

CREATE TABLE field_operator_hst (

fldName VARCHAR(40) NOT NULL COMMENT "Field name",cmpLongName VARCHAR(200) NOT NULL COMMENT "Company name",fldOperatorFrom DATETIME NOT NULL,fldOperatorTo DATETIME,fldNpdidField INTEGER NOT NULL COMMENT "NPDID field",cmpNpdidCompany INTEGER NOT NULL COMMENT "NPDID company",fldOperatorDateUpdated DATETIME COMMENT "Date updated", dateSyncNPD DATETIME NOT NULL, PRIMARY KEY (fldNpdidField, cmpNpdidCompany, fldOperatorFrom, fldOperatorTo)) ENGINE=InnoDB DEFAULT CHARSET=utf8 COMMENT "Field: Operators";ALTER TABLE f ield $_{o}$ perator $_{h}$ stADDFOREIGNKEY(cmpN pdidCompany)REFERENCES company(cmpN pdidCompany); ££ALTERTABLE field perator $_{h}$ stADDFOR

Figure 7: field_operator_hst table definition

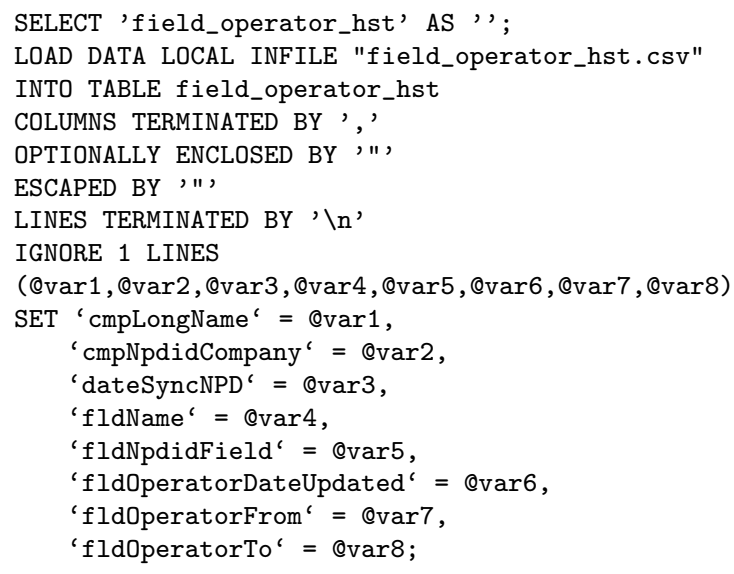

Figure 8: Script for loading the field_operator_hst table with data from the CSV file field_operator_hst.csv.

Entity maps. Each entity map that is selected for this lifting step results in a class declaration using the specified URI as identifier, and adding annotations that reflect the labels, description and provenance information. Ontology Rule 1 defines this translation formally. ${ }^{8}$

Applying the rule to the entity map in line 2 of Figure 5a will result in the following axioms:

\section{Declaration(Class(ndpv:Field))}

AnnotationAssertion(rdfs:label ndpv:Field "Field")

AnnotationAssertion(rdfs: comment ndpv:Field

"Fields on the NCS")

Attribute maps. Similarly, attribute maps result in property declarations using the specified property type to control what type to declare. Ontology Rule 2 defines the translation, and applying it to the attribute map in line 1 of Figure $5 \mathrm{c}$, will give the following

${ }^{8}$ To keep the rules simple, they do not include representing provenance information in the ontology. An example of how this is represented is found in Figure 9. axioms:

\author{
Declaration(DataProperty(ndpv: name)) \\ AnnotationAssertion(rdfs: label ndpv:name \\ "Field name")
}

Attribute maps that are flagged as mandatory are translated to unqualified existential restrictions with Ontology Rule 4; each class that refers to the attribute map is set as a subclass of the restriction class expression. The attribute map in line 4 in Figure 5c contains an attribute map where the property type is a datatype property and the mandatory flag is true. This map is referred to by the entity map that produces the class npdv: FieldOperator (line 2 of Figure 5a). The result of applying Ontology Rule 4 to 
this attribute map is the following axioms: ${ }^{9}$

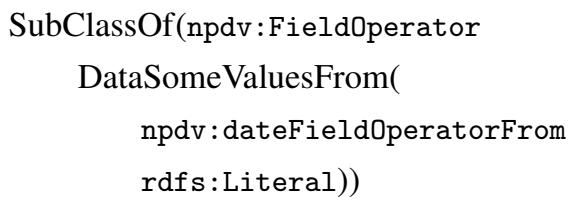

Relation maps. Relation maps are used to transform foreign keys into object properties and restriction axioms. Ontology Rule 5 translates relation maps to qualified existential restrictions. The restriction is qualified with the class specified as the target entity of the relation map, and setting the source entity class as subclass of the restriction class.

The relation map in line 1 of Figure 5d specifies:

- the source entity class as npdv:FieldOperator,

- the target entity class as npdv: Company, and

- the property URI as npdv:fieldOperator.

Applying Ontology Rule 5, this map results in the following axioms: ${ }^{10}$

\author{
Declaration(ObjectProperty(npdv: fieldOperator)) \\ SubClassOf(npdv:Fieldoperator \\ ObjectSomeValuesFrom( \\ npdv:fieldOperator \\ npdv: Company))
}

Identifier maps. Identifier maps are translated to OWL keys with Ontology Rule 3. A key axiom is declared for the class that the identifier map refers to, if all the attributes needed to create the key are also included in the map of the class to be identified.

Applying the rule to the identifier map in line 2 of Figure $5 b$, creates the following axiom:

\section{HasKey(npdv:Field () (npdv:idNPD))}

No key is produced for the identifier map in line 1 of Figure $5 b$ since not all the attribute maps specified for the identifier map (fldNpdidField, cmpNpdidCompany, fldOperatorFrom, fldOperatorTo) occur in the attribute map list (E4) of the entity that is to be identified by the key: (dateSyncNPD, fldOperatorDateUpdated, fldOperatorFrom, fldOperatorTo).

\footnotetext{
${ }^{9}$ In description logic syntax this would naturally translate to the axiom npdv:FieldOperator $\sqsubseteq \exists$ npdv:dateFieldOperatorFrom

${ }^{10}$ In description logic syntax this translates to:
} npdv:FieldOperator $\sqsubseteq \exists$ npdv:fieldOperator .npdv: Company .
Subtype maps. Subtype maps produce in this lifting step subclass axioms from values in designated database columns. This map hence behaves differently than the other maps which use only the database schema information to build ontology constructs. A subtype map contains the necessary information to convert database values into URLs which are declared as subclasses of the class URI specified by the map. The database value is converted to a URI by optionally prepending and appending it with the given prefix and suffix, and also optionally translating the value with a translation table. Translation tables will be explained more thoroughly in next subsection. Ontology Rule 6 formally specifies the translation of subtype maps.

Consider line 1 in Figure 5e; this map specifies that the values of the $f c l K i n d$ of the table that the entity map facility_fixes represents shall be converted to URIs by prepending the prefix npdv: and appended the string Facility. There is no specified translation table for this map, the default transformation set for the use case is to convert the database values to UpperCamelCase strings. Assume "CONDEEP 4 SHAFTS" and "SINGLE WELL TEMPLATE" are the only values in the fclKind column. The Ontology Rule 6 will then produce the following axioms from this subtype map:

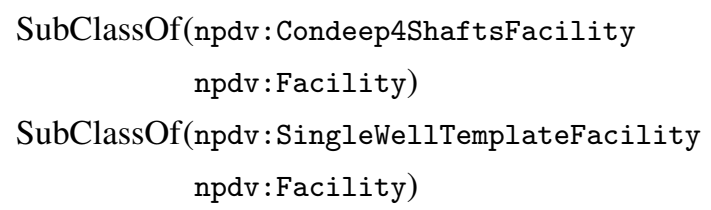

It is worth noting that, unlike the other specification maps that use the information stored in an OBDA specification to produce output, subclass maps use also the actual data contents. Hence, the size of the output produced by subtype maps depends also on the size of the database data contents and not only the schema.

Specification maps are not considered for the lifting step from the database to an ontology if they lack required fields. Specifically, entity maps, attribute maps, and relation maps are not applied if they lack a URI. This is the method used for refraining to transform certain database constructs to ontology constructs. In our running example, this is seen in, for example, the attribute maps in lines 6 and 7 of Figure 5c, which both have unspecified OWL property URI values. These columns are chosen not to be mapped by their respective attribute maps since they are covered by the relation maps in lines 1 and 2 of Figure $5 \mathrm{~d}$.

By keeping track of which classes and datatypes the generated ontology properties relate, we can add domain and range axioms to reflect this. In the current version of the OBDA specification we add an axiom giving the domain of a property whenever the map that produced the property is only referred to by one entity map, i.e., the property uses only one class or datatype as its domain, similarly for the ranges of properties. This can be easily changed to make axioms that set the domain and range of properties to all (or some number of) classes and datatypes that a property relates.

To provide a combined example of many of the abovementioned specification maps, Figure 9 gives an excerpt of the class npdv:Fieldoperator as defined by the map specifications of field_operator_hst in Figure 5, i.e., the entity map 


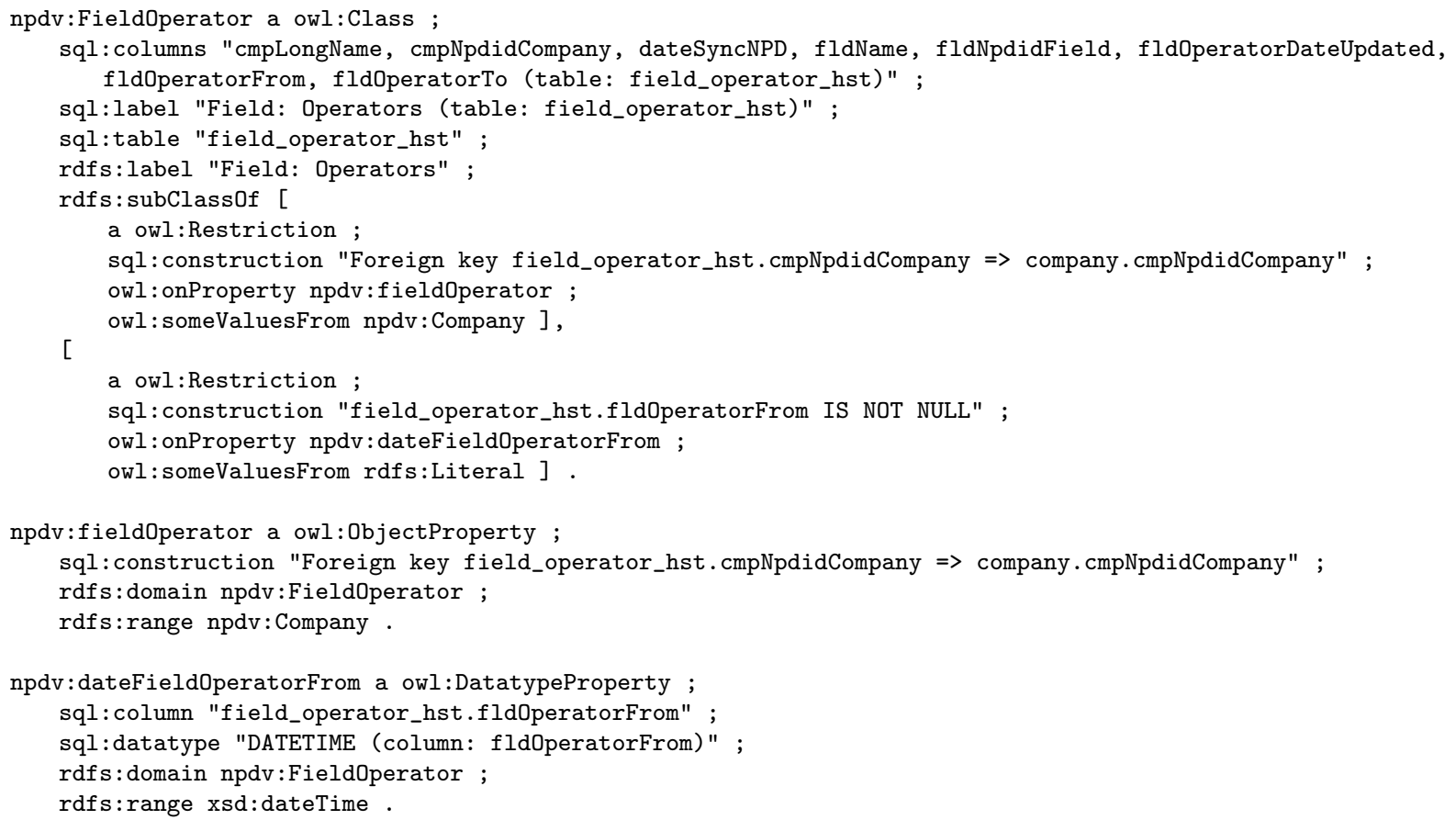

Figure 9: Excerpts of the npdv:FieldOperator class definition.

in line 1 of Figure 5a with referenced attribute maps, and the relation maps in Figure 5d; the result of generating ontology axioms for the columns dateSyncNPD and fldOperatorTo is dropped as they follow the same pattern as for other maps. We note that only the four columns listed in the last field of the entity map: dateSyncNPD, fldOperatorDateUpdated, fldOperatorFrom, fldOperatorTo are mapped, with the effect that the primary key of the table is not represented as an OWL key, since some of the properties necessary to define the key are missing. The foreign key column cmpNpdidCompany is mapped to the object property npdv:fieldOperator and to a qualified existential restriction axiom. The mandatory column fldOperatorFrom is mapped to the datatype property npdv:dataFieldOperatorFrom and to an essentially unqualified existential restriction axiom. The object property npdv:fieldOperator only relates the classes seen in this example, so a domain and range for the property is set, respectively npdv:FieldOperator and npdv:Company. Similarly for the datatype property npdv:dataFieldOperatorFrom, the domain and range is set to npdv:FieldOperator and xsd:dateTime. Lastly, as seen from the sql: prefixed properties, which are taken from a custom-built ontology, the constructed ontology is annotated with the labels and, when available, the descriptions set in the specification. Additionally, as far as possible, each ontology axioms is marked with the database origin of the axiom, for instance naming as source a specific foreign key from the database. We do this so that axioms can easily be traced back to the database, which helps the design process of an OBDA engineering specification.

\subsection{Designing Mappings from the Database to the Ontology}

Given that the schema of the relational database and the resulting ontology are thoroughly described and related by the OBDA engineering specification, the mapping between the two representations is already implicitly defined. What remains is to define how database values are translated to RDF resources, which means specifying URIs and translating data values to the correct format according to its RDF datatype. This is the task of the mappings produced by this transformation step. A mapping consists of two parts, a query over the relational database and a description of how the results of this query is output as RDF triples.

To produce these mappings, all specification maps are used. The relevant parts of the specification are those necessary for building the required database queries and for specifying the RDF output. This means essentially that all fields, except those that contain metadata about the database schema and ontology, for instance, database datatypes and textual descriptions, are relevant. We refer to Figure 4 on page 8 for the selection of these fields, and point out that the fields used exclusively to produce mappings are those which are directly related to specifying RDF output: URI patterns (I3, A8) and other fields that are used for designing proper RDF resources, value translations (A7) and value language tags (A9).

Before we explain the output of the different maps, we explain how URI patterns and translation tables work, as these are central to the production of mappings. Consider the URI pattern in line 1 of Figure 5b. It specifies that URIs are to be built by replacing the special placeholders in the pattern, like $\{\$ 1\}$, with values in the database that are collected from the columns that the attribute maps of the identifier map refer to. The number in the place- 
holder corresponds to the index of the attribute map in the list. This is very similar to the string templates of the R2RML mapping language. Translation tables offer fine-grained translations of database values to RDF resources. This is feature is taken from D2RQ's mapping language [21]. A two-column translation table specifies a partial one-to-one mapping function between the two domains. This feature is very useful for translating sets of values that are otherwise difficult to automatically transform to suitable URIs using generically defined translation functions, or for simple translations that involve few values. An example of the latter from our use case, is the need to translate different two-value value domains, for instance, $\mathrm{YES} / \mathrm{NO}$, in different variations $(\mathrm{Y} / \mathrm{N})$ and languages $(\mathrm{Ja} / \mathrm{Nei})$, and Turnarea included/Turnarea not included, into the standard RDF Boolean values.

We now explain how the specification maps are used to produce mappings.

Entity and identifier maps. Entity and identifier maps are used to specify mappings that create ontology individuals from the rows of database tables; the identifier map specifies the URI of the individuals and the entity map its type. This is formally specified by Mapping Rule 1.

The entity map and identifier map in lines 1 of Figure 5a and 1 of Figure 5b result in the following mapping seen in Figure 10a together with the RDF output produced when the mapping is applied to the data in Figure 6. Note that the date value have been transformed to the correct datatype format.

Attribute maps. Attribute maps specify mappings that relate the individuals that represent the rows of a table to their values in different columns. An attribute behaves slightly differently according to the specified property type of the map. If the property type is a datatype property, then the column value may be typed (with a datatype) or given a language tag. If the type is an object property, then a URI pattern may be used to convert the database value to a proper URI; it is also possible to specify that the database value already is a valid URI. In all cases (datatype, object or annotation property), a translation table can be used to produce correct output from the values in the database. Mapping Rule 2 specifies this transformation.

Applying this rule to the attribute map in line 4 in Figure 5c results in the first mapping seen in Figure 10b, which maps a datatype property; we notice that the mappings set the correct datatype for the object resources of the output triples. Mapping no. 2 in the same figure represents a mapping of a object property where the complete URI is stored in the database; this is the mapping resulting from attribute map in line 12 of Figure 5c. Mapping no. 3 illustrates the mapping resulting from translating a object property pattern mapping, line 13 in Figure 5c. Here, values from the database are combined with the specified URI pattern to form URIs; in this case also using "external" URIs, https://ws.brreg.no/lod/data/\$1.

Relation maps. Relation maps result in mappings that create triples relating individuals created from the relation map's source and target entity maps references. Mapping Rule 3 specifies this transformation.
The relation map in line 2 of Figure $5 d$ will result in the mapping shown in Figure 10c. Notice that the mapping requires that both the sets of columns necessary to construct the URIs to be related are collected, and furthermore that the two database tables (that are already related through a foreign key) must be joined appropriately.

Subtype maps. The outcome of subtype maps in this transformation step is one mapping per subclass axiom that was generated by the subtype map in the transformation step for constructing an ontology from the database, i.e., by Ontology Rule 6. The mapping results in a type relationship between the individual that represents the row of the table that the subtype map is defined for and the subclass created in the ontology for the same row. This is formulated more precisely by Mapping Rule 4 .

Using the same example as for the explanation of subtype maps in previous section, assume "CONDEEP 4 SHAFTS" is a value in the fclKind column found in the subtype map in line 1 in Figure 5e. A mapping for this value is found in Figure 10d.

The materialised triples resulting from using all applicable mappings on the data in Figure 6 is given in Figure 11. ${ }^{11}$ Figures 12 and 13 contain the mappings, respectively in D2RQ's and Ontop's mapping languages, to the parts of the npdv:FieldOperator class in Figure 9.

\subsection{Discussion}

The OBDA specification language is capable of representing common relational database structures and translating these to ontology constructs, together with mappings for transforming data from source the database to the ontology. As explained and exemplified in this section, the language allows for transformations with considerable customisation, many of which are basic, but nevertheless important, in order to produce OBDA systems of high quality. The clear strengths of the language are its design and compact format that support the construction and maintenance of multiple artefacts of an OBDA system. For instance, by limiting the specification of URI patterns to only identity maps and attribute maps, the URIs generated by the mappings created by a specification may be changed easily and consistently in selected and natural parts of the specification. The shortcomings of the specification is its limited support of corner cases, exemplified by the fact that is it not possible to easily specify mappings that require the joining of multiple database tables, or datatype property values that should be produced by combining multiple database columns. In the current version of the specification language such descriptions must be added manually after generating the OBDA artefact.

The specification language is designed to be very general and does not commit to specific versions of schema, ontology or mappings languages, nor to any given ontology engineering methods or reasoning requirements. The current specification implementation outputs the OBDA output to single standalone

\footnotetext{
${ }^{11}$ Note that the RDF data is published in a linked data front-end according to best-practice principles, meaning the data may be examined by visiting the URIs with a web browser.
} 
DB: SELECT fldNpdidField, cmpNpdidCompany, fldOperatorFrom, fldOperatorTo

FROM field_operator_hst

RDF: 〈npd:field/\{fldNpdidField\}/operator/\{cmpNpdidCompany\}/history/\{fldOperatorFrom\}/\{fldOperatorTo\}>

rdf:type npdv:FieldOperator

<npd:field/46437/operator/562/history/1986-12-15T00:00:00/1996-06-19T00:00:00>

rdf:type npdv:FieldOperator

(a) Entity and identifier mapping example

\section{Mapping datatype properties:}

DB: SELECT fldNpdidField, cmpNpdidCompany, fldOperatorFrom, fldOperatorTo FROM field_operator_hst

RDF: 〈npd:field/\{fldNpdidField\}/operator/\{cmpNpdidCompany\}/history/\{fldOperatorFrom\}/\{fldOperatorTo\}> npdv: dateFieldOperatorFrom $\{\text { fldOperatorFrom }\}^{\text {” } x s d: \text { dateTime }}$

<npd:field/46437/operator/562/history/1986-12-15T00:00:00/1996-06-19T00:00:00>

npdv:dateFieldOperatorFrom "1986-12-15T00:00:00" ^^xsd:dateTime

2. Mapping object properties using URI values from the database:

DB: SELECT fldNpdidField, fldFactPageUrl

FROM field

RDF: 〈npd:field/\{fldNpdidField $\}>$ npdv:factPageURL <\{fldFactPageUrl $\}>$

\section{Mapping object properties using URI patterns:}

DB: SELECT cmpNpdidCompany, cmpOrgNumberBrReg FROM company

RDF: 〈npd:company/\{cmpNpdidCompany\}> owl:sameAs <https://ws.brreg.no/lod/data/\{cmpOrgNumberBrReg\}>

(b) Attribute mapping examples

DB: SELECT s.fldNpdidField, s.cmpNpdidCompany, s.fldOperatorFrom, s.fldOperatorTo, t.fldNpdidField FROM field_operator_hst $s$, field $t$ WHERE s.fldNpdidField $=$ t.fldNpdidField

RDF: 〈npd:field/\{s.fldNpdidField\}/operator/\{s.cmpNpdidCompany\}/history/\{s.fldOperatorFrom\}/\{s.fld0peratorTo\}> npdv: operatorForField <npd:field/\{t.fldNpdidField $\}>$

<npd:field/46437/operator/562/history/1986-12-15T00:00:00/1996-06-19T00:00:00> npdv: operatorForField <npd:field/46437>

(c) Relation mapping example

DB: SELECT fclNpdidFacility

FROM facility_fixed

WHERE $\mathrm{fclKind}=$ "CONDEEP 4 SHAFTS"

RDF: 〈npd:facility/\{fclNpdidFacility $\}>$ rdf:type npdv:Condeep4ShaftsFacility

(d) Subtype mapping example

Figure 10: Mapping examples from applying the specification rules to selected specification maps in Figure 5. The RDF triples given in (a), (b) and (c) are the results of using the mappings on the source data in Figure 6. 
<http://sws.ifi.uio.no/data/npd-v2/field/46437/operator/562/history/1986-12-15T00:00:00/1996-06-19T00:00:00> npdv: dateFieldOperatorFrom "1986-12-15T00:00:00" "xsd:dateTime ;

npdv:dateFieldOperatorTo "1996-06-19T00:00:00" “xsd:dateTime ;

npdv:dateSyncNPD "2013-05-08T00:00:00" ^xsd:dateTime ;

npdv:fieldOperator <http://sws.ifi.uio.no/data/npd-v2/company/562> ;

npdv:operatorForField <http://sws.ifi.uio.no/data/npd-v2/field/46437> ;

a npdv:FieldOperator

Figure 11: Materialised RDF of the data in Figure 6

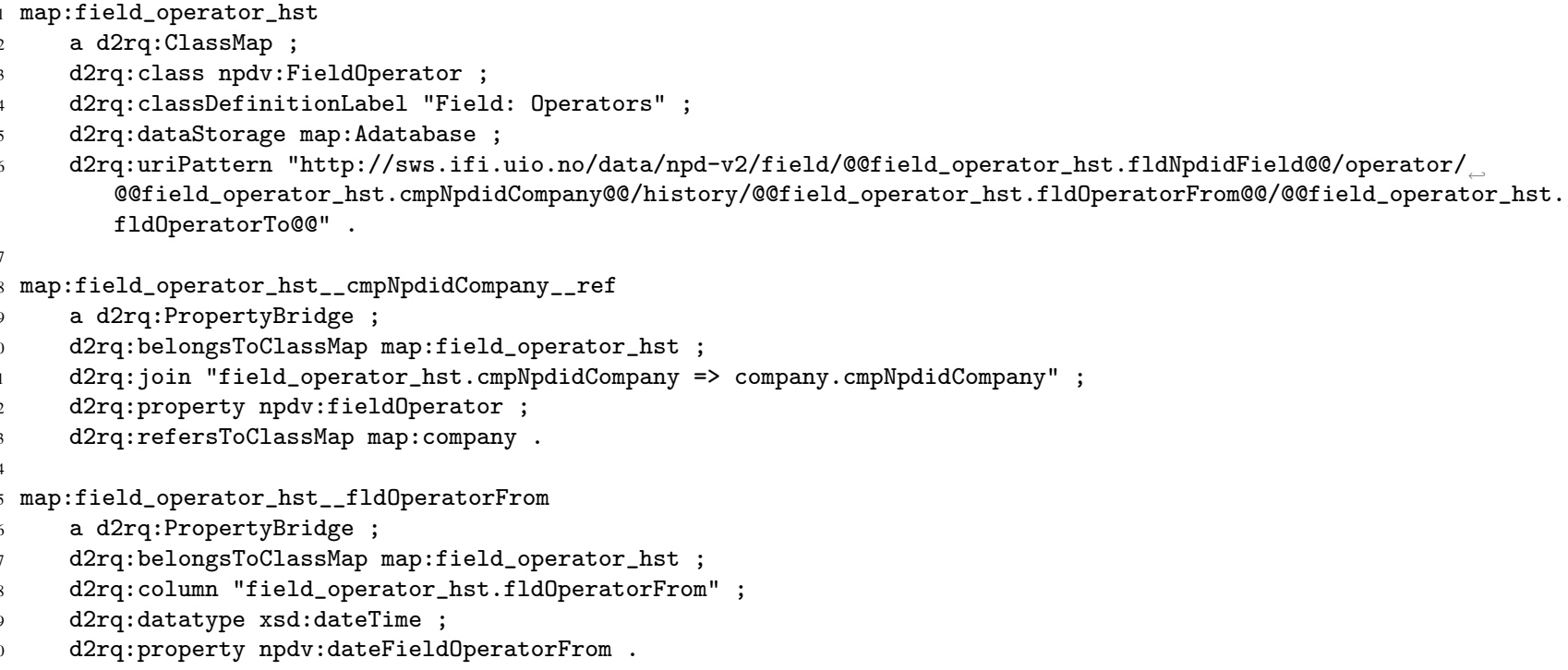

Figure 12: Excerpt of D2RQ mappings from database table field_operator_hst to npdv:FieldOperator and related properties.

mappingId Mapping:00819:Table:npdv:FieldOperator

target npd:field/\{fldNpdidField\}/operator/\{cmpNpdidCompany\}/history/\{fldOperatorFrom\}/\{fldOperatorTo\} a npdv:

FieldOperator .

3 source SELECT fldNpdidField,cmpNpdidCompany,fldOperatorFrom,fldOperatorTo FROM field_operator_hst

mappingId Mapping:00256:Literal:field_operator_hst:fldOperatorFrom

6 target npd:field/\{fldNpdidField\}/operator/\{cmpNpdidCompany\}/history/\{fldOperatorFrom\}/\{fldOperatorTo\} npdv: dateFieldOperatorFrom \{fldOperatorFrom\}" "xsd:dateTime .

source SELECT fldNpdidField, cmpNpdidCompany, fldOperatorFrom, fldOperatorTo FROM field_operator_hst

mappingId Mapping:00901:ForeignKey

target npd:field/\{field_operator_hst.fldNpdidField\}/operator/\{field_operator_hst.cmpNpdidCompany\}/history/\{ field_operator_hst.fldOperatorFrom\}/\{field_operator_hst.fldOperatorTo\} npdv:fieldOperator npd:company/\{company. cmpNpdidCompany\}.

11 source SELECT field_operator_hst.fldNpdidField, field_operator_hst.cmpNpdidCompany, field_operator_hst.fldOperatorFrom, field_operator_hst.fldOperatorTo, company.cmpNpdidCompany FROM field_operator_hst INNER JOIN company ON field_operator_hst.cmpNpdidCompany = company. cmpNpdidCompany

Figure 13: Excerpt of Ontop mappings from database table field_operator_hst to npdv:FieldOperator and related properties. 
files. If a specification should fit particular needs, the output generated from an OBDA specification may be profiled by altering the rules for translating specification instances to OBDA system components.

\section{Use Case Study}

In this section we present the use case study that has guided the development of the OBDA engineering specification and show the applicability of the specification in the development and design of OBDA systems. We also share from our experience of designing and installing multiple different OBDA systems starting only with the data sources, which are on different formats and with little and often poorly structured schema information.

We first present the datasets in our use case, and proceed to explain how an OBDA engineering specification and additional supporting ontology was developed for these datasets. Then we present the specific tools that we used in our study, and how they were used in the process of designing and installing the different OBDA systems. We conclude the section by reporting on the results from using the OBDA specification and tools, and a query evaluation test we performed in order to examine the suitability of the different OBDA architectures and tools.

\subsection{The Use Case Datasets}

The datasets in our case study are the NPD FactPages, the Diskos database and the NORLEX database. They all concern information related to the exploration and production of petroleum on the Norwegian Continental Shelf (NCS).

NPD FactPages. The NPD FactPages [43] contain data about the petroleum activities on the NCS. This means data about the companies that operate on the NCS, e.g., oil companies that operate fields or own shares of production licences, and service companies that perform seismic surveys; geographical data for different physical installations, like platforms, pipelines, and process plants, and the areas of fields and seismic surveys; wellbore data from results of tests taken during drilling; transfers of shares of production licences between companies; and production results measured in volumes of petroleum. The dataset is kept updated to reflect ongoing activity, e.g., the results of exploratory drilling, locations of ongoing seismic surveys, and monthly production figures for the NCS. Additionally, historical data, that is data about shutdown platforms, completed surveys, old production data and more, is also represented in the dataset, some of it dating back to the early 1970s when oil production on the NCS started.

The NPD FactPages are made available as open data [44, 45] in a web application serving a set of HTML pages in a "factsheet format". Most of the background data for a fact page is also available for download in bulk as tabular data in CSV, Excel and XML format. The published dataset is updated daily, and changes to the dataset schema occur at irregular intervals.

A typical use of the dataset is by engineers in oil companies who want to find information related to an area of exploration they are examining. The NPD FactPages, and its accompanying
FactMap application [46], can then be used to identify nearby wellbores and see if there exist test results from these wellbores, or to find other locations that have the same type of reservoir formation that the current area contains, for instance, a promising sand type formation, or simply to establish the ownership of a particular production licence or oil field.

Diskos Database. The Diskos database is the common national data repository for seismic and navigational data, production data and well data from the NCS [47]. This amounts largely to data from different types of seismic surveys, and different types of measurements taken from wellbores, for example rock and core sample tests, and fluid and pressure tests. Unlike the NPD FactPages, where mostly metadata and aggregated data about this type of information is kept, the Diskos database also contains the actual data collected from the surveys and tests; additionally, its metadata is often richer than for the similar NPD FactPages' data. The database contains parts of the NPD FactPages for reference to related data on the NCS.

The Diskos database currently contains 500 TB of data, which is kept in a high-volume data storage capable of handling large amounts of traffic. The data is accessed by users using custombuilt software provided by the current operator of the Diskos database. Using this tool we exported data from different views into 17 CSV files, and additionally downloaded one Excel spreadsheet.

In large oil companies, Diskos is typically used by data managers who are responsible for gathering all the relevant data for a project working on the exploration or development in a particular area. Needless to say, seismic data and well test data is an extremely important asset for petroleum engineers as it is vital for analysing results to discover new deposits of oil and gas.

Norwegian Offshore Stratigraphic Lexicon Database. The Norwegian Offshore Stratigraphic Lexicon (NORLEX) [48] database contains regional lithostratigraphy and biostratigraphy ${ }^{12}$ for the North Sea, Norwegian Sea, Barents Sea and Svalbard, following guidelines laid out in the International Stratigraphic Guide [49]. The database is created and maintained by a project led by the Geology Museum at the University of Oslo, with scientific contributions from the NPD, the Diskos group and major oil companies. The aim of the project is to update outdated lithostratigraphic data previously published by the NPD, amend this information with biostratigraphic data, and compile the results into a relational database to be published online and made accessible through an interactive user interface [48]. The project website and online database interface is available at http://nhm2.uio.no/norlex/.

\footnotetext{
${ }^{12}$ Stratigraphy is the description of all rock bodies forming the Earth's crust and their organisation into distinctive, useful, mappable units, based on their inherent properties or attributes, in order to establish their distribution and relationship in space and their succession in time, and to interpret geologic history. Lithostratigraphic units are bodies of strata based on their lithology, i.e., observable physical features, such as colour, texture, grain size, and composition. Biostratigraphic units are bodies of strata that are defined or characterised on the basis of their contained fossils [49].
} 
The NORLEX database was made available as a MysQL database dump.

\subsection{Constructing an OBDA Engineering Specification}

An OBDA engineering specification is an asset that describes the construction of multiple artefacts of non-trivial complexity. The process of creating a specification is therefore also complex. It is possible to bootstrap a specification from the source data, but as explained in the previous section, not all necessary data is available from the source. We will now explain the steps we took to create the specification.

Lifting Tabular Files to a Database. From Figure 2 it is clear that it is possible to bootstrap information for creating database tables and columns source tabular files. What is not possible to directly read from the tabular files are primary keys and foreign keys. Additionally, not all information required for specifying tables and columns is available from the files; what is missing are labels and textual descriptions, and column datatypes and optional/mandatory flags.

In our case, in order to let the specification bootstrapper correctly read the tabular files, some files needed to be cleaned for irregularities. These where largely due to data values containing linebreaks, hence destroying the structure of the file.

After bootstrapping the specification from the tabular files, textual labels and description were added manually when this information was available (in various formats) from the source data provider. Datatypes and optional flags where set by manually inspecting the data files. We experimented with automatic procedures for setting datatypes and optional flags, but in our experience this was difficult to automate, since the dataset contains too many ill-shaped values that need to be cleaned in order to fit the intended datatype, and available cleaning tools, like OpenRefine [50], where not possible to run as a batch process. This is a requirement in our setup, since loading data and processing the specification is a task that need to be executed regularly. Our cleaning procedure is therefore manually created, by specifying a list of corrections of values and formats to those accepted by the database. Primary and foreign keys were also set by manual inspection, but here field names in many cases provided invaluable help, for instance for the NPD FactPages dataset many keys are prefixed with Npdid (NPD ID).

The added information was tested by iteratively loading the generated SQL schema and tabular data into the newly constructed database, and processing any error messages that the database reported. Thus, the work associated with this transformation step is very technical and focused on syntactical problems of making the tabular data fit in a relational database.

Lifting the Database to an Ontology. As indicated by Figure 2 the by far most laborious part of creating the OBDA specification, is engineering the lifting of the database to the ontology. This is naturally the step that to the largest degree concerns semantic modelling, so knowledge of standard ontology engineering methods, cf. [51], are relevant for this task.

The specification bootstrapper will create a direct mapping specification of the database, with one entity map per table, one identifier map per primary key, one attribute map per column and one relation map per foreign key. The remaining manual tasks are:

- Deciding if there are any bootstrapped specification maps (entity maps, attribute maps or relation maps) that should not be mapped to the ontology.

- Deciding if any tables or columns should have multiple specification maps.

- Selecting the attribute maps to map for each entity map.

- Deciding what type of property columns should be mapped to.

- Identifying columns that contain values that should be handled by subtype maps.

- Specifying URIs for all specification maps.

We discuss these in turn below. Note that these choices are very tightly connected to the production of mappings which we shall explain next.

Cases where it is natural to exclude a specification map from producing output to an ontology, is when the database is oddlyshaped (from the viewpoint of automatic ontology bootstrapping) due to database normalisation or lack of normalisation. A case of the first is when a multivalued attribute is represented with a separate table; from the use case, field_description is a table containing a description over limited number of events for each field. Instead of representing such descriptions with a class, we chose to relate these values by a limited set of properties directly related to the field. In the other case, removing attributes from entity maps can be necessary when the columns are duplicated across multiple tables. In the use case datasets, columns from tables that are referred to are often also included in other tables even though the table is properly referenced using a primary key value, for example the table field_operator_hst in Figure 7 contains the field name (fldName) even though this is also a column in the table field which field_operator_hst refers to by a foreign key. This denormalisation is probably implemented to make the database more user-friendly, but creates an erroneous ontology by connecting a property to the wrong classes.

It is natural to map a table to multiple classes when the table represents more than one concept. From the use case, the wellbore_exploration is an example. This table contains information about exploration wellbores, including information about the stratigraphic layers that the wellbore penetrates. Additionally, the table includes information that is directly associated to the stratigraphic layer, but not the wellbore. In this case we have separated the information about the stratigraphic layer into separate classes, by specifying two entity maps for the wellbore_exploration table, and partitioning the attribute maps over these entity maps.

When specifying subtype maps, important considerations are how to generate URIs from the values that represent subclasses. In many cases using a translation table is a good choice, as it gives detailed control of the translation, for instance when 
values are to be translated to class URIs defined in existing ontologies. The downside of using translation tables is that the translation table needs to be kept updated to reflect the values in the corresponding database column.

Since a URI can and must be specified for every specification map where this is possible, it is easy to design your own ontology vocabulary or use an existing vocabulary when lifting the database to an ontology.

Specifying Mappings from the Database to the Ontology. As with the lifting of the tabular files to a database, this step is also technical in character as it concerns the problem of "fitting" database values into RDF format. The tasks that are necessary in this step are:

- Designing URI patterns.

- Setting correct datatypes or language tags.

- Possibly translating values with translation tables.

Designing a consistent set of URI patterns can increase the semantic value of the resulting URIs. As a case in point, we specified informative URI patterns which often were built by extending existing URI patterns, e.g., three URIs for a wellbore, a wellbore core and a wellbore core photo, are

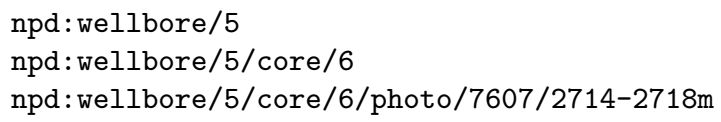

The structure of these URIs indicate a hierarchical relationship between the instances they represent and may help a user better understand the dataset. There are however issues with including information in URIs; for general discussions on how to design proper URIs, see [52, 53].

Specifying datatypes for datatype property values are usually not needed, since the translation of the common SQL datatypes to RDF datatypes is set by the translation scripts. However, for less common datatypes this may be necessary. In the use case, the datasets contained geometric values stored as a GEOMETRY datatype in the database. These values were represented as RDF by using datatypes from the GeoSPARQL ontology [54].

We used translation tables to translate database values to URIs in order to represent geologic periods, which are named with string such as CRETACEOUS, TRIASSIC, LATE JURASSIC in the database, using the International Chronostratigraphic Chart (2012) (ICS) ontology [55], which models the global time scale and represents these eras as individual URIs.

\subsection{Further Ontology Development}

Although the ontology that is produced by the engineering process is an significant improvement over a direct mapping generated ontology, this technique cannot in general replace manual ontology development. There will be information that is difficult to extract from the database and also simpler to include in the ontology using a regular ontology editor, rather than adding such capabilities in the specification language.

In our case, we extended the ontology by manually adding axioms which capture information that was not available to the automatic process. This was largely done to adding atomic general superclasses to the generated classes, e.g., npdv:Facility was set as the superclass of the generated classes npdv:FixedFacility and npdv:MoveableFacility, and introducing a set of mutually disjoint top level classes like npdv: Agent, npdv: Area and npdv: Point. A selection of classes and properties that model geographical data were mapped to the GeoSPARQL vocabulary and to the ICS vocabulary.

The manually constructed ontologies were kept in separate files that represent separate domains: geology, geometry, facilities (off- and onshore constructions), organisational, petroleum production and seismic surveys. In order to ensure a minimal quality of the integration of the different ontologies, all classes where placed under an appropriate class from the upper ontology Basic Formal Ontology BFO [40]. Only for the NPD FactPages dataset did we do a thorough manual ontology engineering effort. The ontologies created for each data source were imported to a "hub" ontology containing only import statements. The three data source hub ontologies were imported to a central hub ontology called NCS. The import relationships between the ontologies are illustrated in Figure 14.

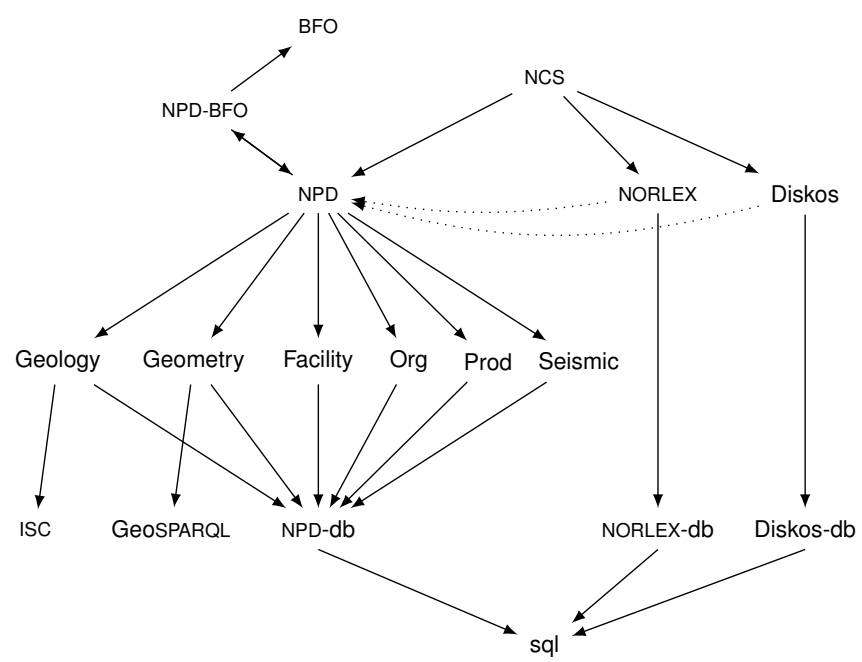

Figure 14: Import hierarchy of ontologies. Ontologies suffixed with $-\mathrm{db}$ are lifted from the databases with the OBDA specification. BFO, ICS and GeoSPARQL are external ontologies, while the others are manually constructed. NPD-BFO contains a mapping from NPD to BFO using subclass axioms. sql contains the annotation properties used by the ontology rule of the specification.

\subsection{Use Case Tools}

As the primary OBDA tools in our case study we have used Ontop [26] version 1.9, Fuseki [31] version 1.0.0, D2RQ [21] version 0.8.1, and Stardog [32] version 2.0. These are further described below. The relational database we used in our study was MySQL version 5.1.

Fuseki. Fuseki is a SPARQL server that is part of Apache Jena, a "free and open source Java framework for building Semantic Web and Linked Data applications." [31] The standalone server 
version (which is the version we used) is bundled with the RDF store TDB, also a component of Jena. In addition, Jena supports storing data in a relational database (SDB) or in-memory. Querying the data is done using the query engine ARQ. Fuseki provides an implementation of SPARQL 1.1 over HTTP, using ARQ.

Stardog. Stardog [56] is a commercial RDF database developed by Clark \& Parsia and "performs reasoning in a very lazy and late-binding fashion: it does not materialise inferences; but, rather, reasoning is performed at query time according to a given reasoning level. This allows for maximum flexibility while maintaining excellent performance and scalability." [32]

D2RQ. D2RQ [57] was initially developed by the Freie Universität Berlin and is "a system for accessing relational databases as virtual, read-only RDF graphs." [21] The relationship between the relational database and the $R D F$ view is given by a set of mappings, similar to those of Ontop. RDF triples are not stored in a triple store; rather, D2RQ transforms incoming SPARQL queries to SQL queries over the relational data. D2RQ does not provide ontological reasoning capabilities.

Ontop. Ontop [58, 59] is developed at the University of BozenBolzano. Query execution in Ontop is based on two rewriting steps, as illustrated in Figure 1b; the user inputs a SPARQL query $q$, which is rewritten to $q_{0}$ using the ontology. $q_{0}$ is then unfolded into the SQL query $q_{\mathrm{DB}}$ using the mappings. The latter query is passed to the database. $q_{\mathrm{DB}}$ is constructed such that the answer from the database is already a serialisation of the triples in the answer, and does not need any further processing before being passed back to the user. This is a high level overview leaving out many details, in particular, many features and optimisations to facilitate faster query answering.

In summary, Fuseki and Stardog provide a physical triple store which require that the source data is transformed into RDF, while Ontop and D2RQ are based on a virtual triple store and transform SPARQL queries into SQL queries which are evaluated over a relational database. Stardog and Ontop support ontological reasoning and both use rewrite techniques for answering queries. Since Ontop relies on query rewriting for the transformation process, reasoning support is restricted to the OWL 2 QL profile. Stardog, on the other hand, supports all owL 2 profiles, but guarantees only complete answers for OWL 2 QL ontologies.

\subsection{Technical Setup of the Use Case Study}

The overall goal of our use case study is to install, design and test multiple OBDA systems that efficiently accesses all the selected data sources, and in the process developing and using the OBDA engineering specification. In this section we will report from many of the specific problems that we were faced with when lifting and representing the source data in different formats: a relational database, an ontology, and mappings, but also problems that were due to the specific formats and tools used in the study.

As discussed in the introduction and illustrated in Figure 1, two important choices when designing an OBDA system is deciding on the type of transformation, if the system shall materialise the data as RDF (data transformation) or leave the data in the original sources and translate queries over the ontology to queries over the database (query transformation), and if the system shall support ontological reasoning. In our case study we have installed instances of all combinations for the two choices:

1. Data transformation and no reasoning. The main tools involved were D2RQ for transforming the data, and Fuseki as triple store and query processor.

2. Data transformation and reasoning support. The main tools were D2RQ for transforming the data, and Stardog as triple store and reasoning enabled query processor.

3. Query transformation and no reasoning support. The main tools were D2RQ for query transformation and MySQL as underlying relational database.

4. Query transformation and reasoning support. The main tools were Ontop for query transformation and query rewriting-based reasoning and MySQL as underlying relational database.

The development and deployment process for the OBDA specification for and on these architectures as it was performed in our use case study is illustrated in Figure 15. These steps were:

1. Downloading data.

2. Bootstrapping the OBDA engineering specification.

3. Creating and loading the relational database.

4. Generating mappings.

5. Materialising data as RDF.

6. Developing the ontology.

7. Loading the systems with data, ontology and/or mappings.

After an initial download of data and the bootstrapping of an OBDA specification, the specification was active in steps 1, 3, 4 and 6: Since the filenames of the CSV files are represented in the specification, this information was used to script an automatic download of the CSV data. The bootstrapping of the specification was only performed once. Steps 3, 4 and 6 was implemented as already explained in this section. Step 5 was performed by the export capabilities of D2RQ, while in the last step the different import features of the individual OBDA tools were used.

These seven steps were not performed in a single straight sequence, but with longer and longer iterative development cycles adding more information and features to the specification. These cycles can be categorised in four:

1. Creating and loading tabular files into the database.

2. Designing RDF output.

3. Ensuring that the produced artefacts loaded into the system.

4. Further ontology development. 


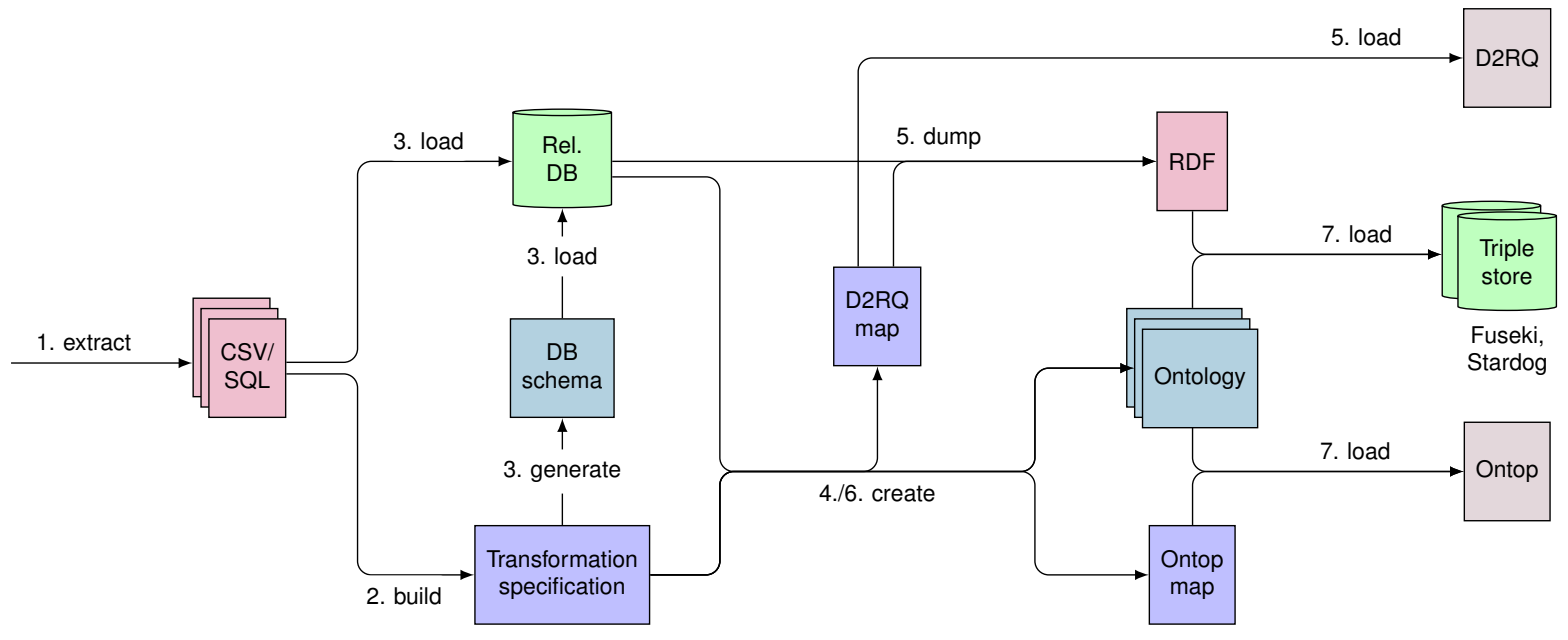

Figure 15: Schematic overview of the deployment process.

In this process the OBDA specification was key in ensuring that all artefacts were synchronised, for instance:

- All URIs are synchronised in all representations.

- Values used to build individual URI are always taken from primary keys.

- Other values that form part of URIs are indexed by the database to make query answering more efficient.

- All URI patterns may be developed in a single specification.

- Mappings, which are very verbose and difficult to manually edit, are automatically generated.

\subsection{Key Characteristics of the Use Case Configuration}

The input and output of the different conversion steps are summarised in numbers in Tables 1 and 2. Table 1 shows in numbers how the input data evolve into mappings and classes and properties through the conversion process. A total of $87 \mathrm{CSV}$ files, one spreadsheet and one relational database was input as source data to the process. This was converted into a relational database with 107 tables and 1,435 columns. To materialise the database as RDF, a D2RQ map for each of the data sources was generated and used to dump the datasets to RDF files containing respectively $2 \mathrm{M}, 5 \mathrm{M}$ and $22 \mathrm{M}$ triples using approximately 4.5 hours, 2.5 hours and 10 hours for the NPD FactPages, NORLEX database and the Diskos data.

The numbers in Table 1 reveal that the schema of the source data is faithfully represented in the relational database, as seen by the fact that the number of CSV files equals the number of tables and CSV fields are almost the same as the number of SQL columns. The added columns are auto increment-valued columns that are created in the absence of a suitable primary key for some tables. Additionally, the increase from number tables to number of D2RQ class maps shows that more than one class per table is created. There are fewer D2RQ columns, due to the effort of removing duplicated unnormalised data in the mapping of the database. Furthermore, that there are more RDF classes than D2RQ class maps shows that some maps generate classes. The difference between D2RQ property maps and RDF properties is explained by the fact that many tables contain columns with the same name, and also some columns with different names are mapped to the same property, e.g., fldName (field name) and cmpLongName (company name) are both mapped to npdv : name. Finally, the numbers for Ontop maps versus D2RQ maps show that Ontop mappings are more verbose than D2RQ's.

It is evident from the number of generated triples that the NPD FactPages dataset is the smallest of the three data sources. However, it is the most complex in terms of the number of classes and properties, containing more than half of the total number of classes and properties generated from all of the data. The Diskos database is by far the largest dataset, giving almost $75 \%$ of the total number of $29 \mathrm{M}$ triples.

Table 2 lists the numbers of axioms, classes, properties and individuals and the expressivity of the ontologies developed in our case study, using data as reported by Protégé 4.3. ${ }^{13}$ The table is grouped according to dataset. Central "hub" ontologies, i.e., npd, norlex, diskos and ncs, which (indirectly) import all ontologies in our study, are listed both with and without imports, and with and without external imports. The norlex and diskos ontologies do not import external ontologies.

The numbers in Table 2 show, as also seen in Table 1, that the ontology for the NPD FactPages is the largest. Furthermore, we see that the generated ontologies $(*-\mathrm{db})$ contribute to the largest numbers, disregarding the external imports, and that their expressivity is consistently $\mathcal{A} \mathcal{L} \mathcal{E}(D)$, as expected from our translation of database constraints to ontological axioms. We notice from the expressivity column that the handcrafted ontologies are not very expressive; they all have the lowest expressivity reported by Protégé, $\mathcal{A} \mathcal{L},{ }^{14}$ except for: npd-geology, which contains a few disjointness axioms; npd-geology, which contains an

13

${ }^{14}$ Although the expressivity reported by Protégé can be an important and valuable characteristic of an ontology, it is not fine-grained enough to positively identify if an ontology fits a specific OWL profile. $\mathcal{A L}$ is used as the base 
Table 1: Conversion process results in numbers

\begin{tabular}{|c|c|c|c|c|}
\hline & NPD & NORLEX & Diskos & Total \\
\hline CSV files & 70 & - & $17+1^{\mathrm{a}}$ & - \\
\hline SQL tables & 70 & 19 & 18 & 107 \\
\hline D2RQ class maps & 80 & 28 & 22 & 125 \\
\hline RDF generated classes ${ }^{b}$ & 137 & 24 & 39 & 195 \\
\hline OWL generated classes ${ }^{\mathrm{c}}$ & 139 & 22 & 36 & 197 \\
\hline CSV fields (distinct) & 963 & - & 306 & - \\
\hline SQL columns & 974 & 151 & 310 & 1,435 \\
\hline D2RQ property maps & 869 & 181 & 338 & 1,383 \\
\hline RDF generated properties ${ }^{b}$ & 337 & 104 & 189 & 626 \\
\hline OWL generated properties ${ }^{\mathrm{c}}$ & 321 & 108 & 199 & 624 \\
\hline Ontop maps & 1,188 & 175 & 344 & 1,707 \\
\hline RDF triples & $2,002,556$ & $5,389,793$ & $21,924,435$ & $29,316,784$ \\
\hline Time to produce, hours & $\approx 4.5$ & $\approx 2.5$ & $\approx 10$ & $\approx 17$ \\
\hline
\end{tabular}

Table 2: Ontology metrics

\begin{tabular}{|c|c|c|c|c|c|c|c|}
\hline Ontology & Axioms & $\begin{array}{l}\text { Logical } \\
\text { axioms }\end{array}$ & Classes & $\begin{array}{r}\text { Object } \\
\text { properties }\end{array}$ & $\begin{array}{l}\text { Datatype } \\
\text { properties }\end{array}$ & Individuals & Expressivity \\
\hline sql & 7 & 0 & 0 & 0 & 0 & 0 & - \\
\hline $\mathrm{npd}-\mathrm{db}$ & 4,103 & 1,069 & 142 & 92 & 225 & 0 & $\mathcal{A} \mathcal{L} \mathcal{E}(D)$ \\
\hline npd-facility & 24 & 7 & 9 & 0 & 0 & 0 & $\mathcal{A} \mathcal{L}$ \\
\hline npd-geology & 23 & 8 & 9 & 2 & 0 & 0 & $\mathcal{A} \mathcal{L} C$ \\
\hline npd-geometry & 38 & 18 & 18 & 2 & 1 & 1 & $\mathcal{A} \mathcal{L} \mathcal{I}$ \\
\hline npd-org & 72 & 17 & 33 & 1 & 0 & 0 & $\mathcal{A} \mathcal{L}$ \\
\hline npd-prod & 83 & 17 & 25 & 2 & 0 & 0 & $\mathcal{A} \mathcal{L}$ \\
\hline npd-seismic & 4 & 0 & 1 & 0 & 0 & 0 & - \\
\hline npd-bfo & 64 & 62 & 75 & 0 & 2 & 0 & $\mathcal{A} \mathcal{L}$ \\
\hline npd & 2 & 1 & 0 & 0 & 1 & 0 & $\mathcal{A} \mathcal{L} \mathcal{F}(D)$ \\
\hline$n p d^{a}$ & 4,649 & 1,278 & 227 & 106 & 226 & 1 & $\mathcal{A} \mathcal{L} C \mathcal{H} \mathcal{I F}(D)$ \\
\hline $\mathrm{npd}^{\mathrm{b}}$ & 17,704 & 8,208 & 344 & 148 & 237 & 855 & $\mathcal{S H O I F}(D)$ \\
\hline norlex-db & 701 & 173 & 25 & 9 & 97 & 0 & $\mathcal{A} \mathcal{L} \mathcal{E}(D)$ \\
\hline norlex & 109 & 39 & 42 & 0 & 1 & 6 & $\mathcal{A} \mathcal{L} C(D)$ \\
\hline norlex ${ }^{a, b}$ & 815 & 212 & 53 & 9 & 97 & 6 & $\mathcal{A} \mathcal{L} C(D)$ \\
\hline diskos-db & 1,395 & 417 & 38 & 6 & 188 & 0 & $\mathcal{A} \mathcal{L} \mathcal{E}(D)$ \\
\hline diskos & 3 & 3 & 6 & 0 & 0 & 0 & $\mathcal{A} \mathcal{L}$ \\
\hline $\operatorname{diskos}^{\mathrm{a}, \mathrm{b}}$ & 1,405 & 420 & 41 & 6 & 188 & 0 & $\mathcal{A} \mathcal{L} \mathcal{E}(D)$ \\
\hline ncs & 0 & 0 & 0 & 0 & 0 & 0 & - \\
\hline$n c s^{a}$ & 6,855 & 1,910 & 308 & 119 & 511 & 7 & $\mathcal{A} \mathcal{L} C \mathcal{H} O \mathcal{I F}(D)$ \\
\hline$n c s^{b}$ & 19,910 & 8,840 & 425 & 161 & 522 & 861 & $\mathcal{S H O I F}(D)$ \\
\hline
\end{tabular}

Metrics given with no ontology imports, unless noted otherwise.

a Only local imports

b All imports 
inverse object property; norlex, which contains intersection axioms; and npd, which contains one functional data property.

All data files, mappings and ontologies from the conversion procedure, except those concerned with the Diskos data, which is not publicly open data, are published on the website http://sws.ifi.uio.no/project/npd-v2/. This includes the NPD FactPages CSV files, the NORLEX database dump, the combined database dump of the two datasets, RDF dumps, D2RQ maps, Ontop maps and the developed ontology. The NPD FactPages and NORLEX data are also published as linked open data at http://sws.ifi.uio.no/data/npd-v2 and http: //sws.ifi.uio.no/data/norlex, respectively.

\subsection{Query Evaluation Experiment}

The OBDA systems in our case study were evaluated by querying the systems with a series of SPARQL queries. In this section we describe the concrete setup of this evaluation.

Queries. Our evaluation consists of a total of 42 SPARQL queries. 21 of these were constructed by translating questions provided by domain expert users of the original datasets into SPARQL queries. These queries are listed in B.1 together with the original domain questions. The questions can be grouped into three categories: Production Licences, Fields and Wellbores. All of the questions concern in part the NPD FactPages, which is natural, since this data source covers all of the category subjects, while the Diskos dataset and NORLEX are mainly only concerned with the category Wellbores. For this reason, only Wellbore queries make explicit references also to other datasets than the NPD FactPages.

The other queries in our evaluation were designed to test and compare the usability of the systems with respect to efficiency and specific language support, e.g., support for various SPARQL query constructs, and test how different types of reasoning configurations and the use of ontological constructs can affect what answers are returned by the systems. For this reason the queries are mostly small and focused; we call these queries, listed in B.2, unit test queries.

We recorded the time it took for the queries to return results, and also counted the number of results returned by each tested OBDA system for each of the queries. The SPARQL queries were often constructed so that they answer the intended question in a more general manner, usually returning more results, and we often use DISTINCT when this is necessary to make sure that the number of answers returned by different systems are comparable.

Setup. We issued the queries over HTTP and measured the time for the systems to return results by the help of the statistics programming language $\mathrm{R}$ [60] and the package RCurl [61]; RCurl an interface to cURL [62], a well-known data transfer tool. The results of the query evaluation experiment are listed in Tables 3 and 4; Table 3 contains the results from the domain expert queries, and Table 4 the unit test queries. For each query we give the number of returned results and the time it took for

language, and all other languages Protégé can recognise are extensions of this, hence it is merely an upper bound, meaning that the ontology is expressed within that language. all the different OBDA configurations: Fuseki, Stardog without reasoning enabled, D2RQ, Ontop, and Stardog with respectively OWL 2 QL, RDFS, OWL 2 EL, and OWL 2 RL reasoning enabled. The timeout on each query was 5 minutes.

For each OBDA configuration, the query evaluation process is as follows. First the order of the 42 queries is randomised, then each query is run against the endpoint using RCurl. This is repeated 25 times. The times listed are the median value of the 25 values, and given in number of seconds. Ontop was set up using the Jetty webserver packaged with the Sesame Workbench as published on the Ontop website [26], configured to use a maximum of 8 GB memory. D2RQ was deployed on a Tomcat server, given a total of 4 GB. Fuseki and Stardog were executed with their self provided server scripts, but given respectively 4 and 6 GB of memory.

Disclaimer. In the case of D2RQ, the configuration of the system in our evaluation is loaded with a map containing the URIPATTERN bug fix, as described in [63]. This means that the URIs produced by the system are incorrect, and thus the number of results returned may very well not be correct according to what a correct intended mapping would give. However, we believe that in those cases where the pattern of the URI is used only to return the results of the correct format (and, e.g., not to match with resources generated from multiple class maps), D2RQ will return the correct number of results. We do not use a mapping that would return correct URIs since initial testing revealed that this map severely hampered query execution and left the system unresponsive for most queries in our evaluation. For this reason we will not further evaluate the query results returned by D2RQ, other than presenting the numbers of answers returned and the time it took together with the other systems in Tables 3 and 4.

The query evaluation experiment was performed in 2013. Since then, Fuseki, Stardog and Ontop have released new versions which may include more query language and reasoning support. We refer the reader to the tool's webpages for more information.

\subsection{Query Evaluation Results}

We now present the results from our query evaluation. We first consider the results from the queries constructed from domain expert questions, then the unit test queries.

Domain queries. As general remarks, we observe that for all these queries, if the query returns any answers from a system, then the number of results is the same as for the other systems that return answers for the same query. This conformity of answers might be surprising at first-why does reasoning not result in added answers? However, on inspection of the SPARQL queries, it is clear that the queries use only vocabulary that is generated from the data sources and not vocabulary from the manually constructed ontology. Since the manually constructed part of the ontology consists mostly of adding superclasses to the generated part, it is reasonable to assume that the manually added ontology is a conservative extension [64] of the generated part, such that reasoning over the whole ontology will not add any additional query answers. Given that all systems deliver the 
Table 3: Query evaluation results I: Domain expert queries

\begin{tabular}{|c|c|c|c|c|c|c|c|c|}
\hline Query & Fuseki & Stardog (SD) & $\mathrm{D} 2 \mathrm{RQ}$ & Ontop & $\mathrm{SD}+\mathrm{QL}$ & SD+RDFS & $\mathrm{SD}+\mathrm{EL}$ & $\mathrm{SD}+\mathrm{RL}$ \\
\hline \multirow[t]{2}{*}{ Query 1} & 2,853 & 2,853 & 2,853 & $-^{a}$ & 2,853 & 2,853 & 2,853 & 2,853 \\
\hline & 53.4 & 0.7 & 20.2 & & 0.9 & 0.7 & 1.1 & 0.7 \\
\hline \multirow[t]{2}{*}{ Query 2} & 910 & 910 & 910 & 0 & 910 & 910 & 910 & 910 \\
\hline & 4.8 & 0.2 & 2.4 & 0.0 & 0.3 & 0.2 & 0.6 & 0.2 \\
\hline \multirow[t]{2}{*}{ Query 3} & 910 & 910 & 910 & 910 & 910 & 910 & 910 & 910 \\
\hline & 0.2 & 0.2 & 0.8 & 0.2 & 0.3 & 0.2 & 0.5 & 0.2 \\
\hline \multirow[t]{2}{*}{ Query 4} & 400 & 400 & 400 & 0 & 400 & 400 & 400 & 400 \\
\hline & 0.1 & 0.1 & 0.3 & 0.0 & 0.2 & 0.1 & 0.4 & 0.1 \\
\hline \multirow[t]{2}{*}{ Query 5} & 17,254 & 17,254 & 17,254 & 17,254 & 17,254 & 17,254 & 17,254 & 17,254 \\
\hline & 4.4 & 2.8 & 238.6 & 3.4 & 3.0 & 2.9 & 3.1 & 2.9 \\
\hline \multirow[t]{2}{*}{ Query 6} & 412 & 412 & 412 & 0 & 412 & 412 & 412 & 412 \\
\hline & 0.3 & 0.1 & 1.8 & 0.0 & 0.3 & 0.1 & 0.9 & 0.1 \\
\hline \multirow[t]{2}{*}{ Query 7} & 101 & 101 & 101 & 101 & 101 & 101 & 101 & 101 \\
\hline & 0.1 & 0.0 & 0.3 & 0.1 & 0.1 & 0.0 & 0.4 & 0.1 \\
\hline \multirow[t]{2}{*}{ Query 8} & 88 & 88 & $-^{\mathrm{b}}$ & $-^{a}$ & 88 & 88 & 88 & 88 \\
\hline & 6.5 & 0.1 & & & 0.4 & 0.2 & 0.9 & 0.2 \\
\hline \multirow[t]{2}{*}{ Query 9} & 88 & 88 & 88 & 0 & 88 & 88 & 88 & 88 \\
\hline & 0.3 & 0.1 & 57.6 & 0.0 & 0.2 & 0.1 & 0.5 & 0.1 \\
\hline \multirow[t]{2}{*}{ Query 10} & 29 & 29 & 0 & 0 & 29 & 29 & 29 & 29 \\
\hline & 0.1 & 0.0 & 0.0 & 0.0 & 0.1 & 0.0 & 0.4 & 0.0 \\
\hline \multirow[t]{2}{*}{ Query 11} & 45 & 45 & 0 & 0 & 45 & 45 & 45 & 45 \\
\hline & 0.1 & 0.0 & 0.0 & 0.0 & 0.2 & 0.1 & 0.4 & 0.1 \\
\hline \multirow[t]{2}{*}{ Query 12} & 1,106 & 1,106 & 0 & 0 & $-^{\mathrm{b}}$ & $-^{b}$ & $-^{\mathrm{b}}$ & $-^{\mathrm{b}}$ \\
\hline & 0.4 & 0.2 & 0.0 & 0.0 & & & & \\
\hline \multirow[t]{2}{*}{ Query 13} & 1,090 & 1,090 & 0 & 1,090 & 1,090 & 1,090 & 1,090 & 1,090 \\
\hline & 0.2 & 0.2 & 0.0 & 1.0 & 0.2 & 0.2 & 0.5 & 0.1 \\
\hline \multirow[t]{2}{*}{ Query 14} & 3,452 & 3,452 & 0 & 0 & 3,452 & 3,452 & 3,452 & 3,452 \\
\hline & 5.7 & 1.2 & 0.1 & 0.0 & 1.3 & 1.1 & 1.6 & 1.3 \\
\hline \multirow[t]{2}{*}{ Query 15} & 29 & 29 & 0 & 0 & 29 & 29 & 29 & 29 \\
\hline & 0.5 & 0.1 & 0.0 & 0.0 & 1.8 & 1.6 & 2.1 & 0.3 \\
\hline \multirow[t]{2}{*}{ Query 16} & 186 & 186 & 0 & 0 & 186 & 186 & 186 & 186 \\
\hline & 0.2 & 0.1 & 0.0 & 0.0 & 0.3 & 0.1 & 0.6 & 0.1 \\
\hline \multirow[t]{2}{*}{ Query 17} & 420 & 420 & 0 & 420 & 420 & 420 & 420 & 420 \\
\hline & 0.3 & 0.1 & 0.0 & 0.6 & 0.2 & 0.1 & 0.5 & 0.1 \\
\hline \multirow[t]{2}{*}{ Query 18} & 35 & 35 & 0 & 0 & 35 & 35 & 35 & 35 \\
\hline & 26.4 & 0.2 & 0.0 & 0.0 & 0.3 & 0.2 & 0.5 & 0.2 \\
\hline \multirow[t]{2}{*}{ Query 19} & 454 & 454 & 0 & 0 & 454 & 454 & 454 & 454 \\
\hline & 2.0 & 0.3 & 0.0 & 0.0 & 0.3 & 0.2 & 0.6 & 0.2 \\
\hline \multirow[t]{2}{*}{ Query 20} & 0 & 0 & 0 & 0 & 0 & 0 & 0 & 0 \\
\hline & 0.3 & 0.8 & 0.0 & 0.0 & 1.5 & 1.4 & 1.9 & 1.4 \\
\hline \multirow[t]{2}{*}{ Query 21} & $-^{b}$ & 68 & 0 & 0 & 68 & 68 & 68 & 68 \\
\hline & & 1.2 & 0.0 & 0.0 & 5.2 & 5.0 & 5.5 & 5.1 \\
\hline
\end{tabular}

The number of results returned by the system for the given query (in bold), and below, the time in seconds.

${ }^{a}$ Error message: Error, the translation of the query generated 0 rules. This is not possible for any SELECT query (other queries are not supported by the translator). Probable cause: query contains a subquery with a variable bound by the outer query.

b Timeout: System does not return within 5 minutes. 
Table 4: Query evaluation results II: Unit test queries

\begin{tabular}{|c|c|c|c|c|c|c|c|c|}
\hline Query & Fuseki & Stardog (SD) & $\mathrm{D} 2 \mathrm{RQ}$ & Ontop & $S D+Q L$ & $\mathrm{SD}+\mathrm{RDFS}$ & $\mathrm{SD}+\mathrm{EL}$ & $\mathrm{SD}+\mathrm{RL}$ \\
\hline \multirow[t]{2}{*}{ Query 22} & 7,441 & 7,441 & 7,264 & 7,312 & 7,441 & 7,441 & 7,441 & 7,441 \\
\hline & 1.0 & 0.8 & 19.9 & 1.0 & 0.8 & 0.9 & 0.9 & 0.9 \\
\hline \multirow[t]{2}{*}{ Query 23} & 0 & 0 & 0 & 1,435 & 1,435 & 1,435 & 1,435 & 1,435 \\
\hline & 0.1 & 0.0 & 0.0 & 89.5 & 10.5 & 10.3 & 11.3 & 10.9 \\
\hline \multirow[t]{2}{*}{ Query 24} & 13,436 & 13,436 & 13,635 & $-^{c}$ & 13,436 & 13,436 & 13,436 & 13,436 \\
\hline & 4.0 & 3.4 & 150.5 & & 3.6 & 3.5 & 3.4 & 3.5 \\
\hline \multirow[t]{2}{*}{ Query 25} & 0 & 0 & 0 & $-^{c}$ & 6,900 & 6,900 & 6,900 & 6,900 \\
\hline & 0.0 & 0.0 & 0.0 & & 1.3 & 1.2 & 1.2 & 1.3 \\
\hline \multirow[t]{2}{*}{ Query 26} & 0 & 0 & 0 & 13,176 & 6,900 & 6,900 & 6,900 & 6,900 \\
\hline & 0.0 & 0.0 & 0.0 & 1.9 & 0.9 & 0.9 & 0.9 & 1.0 \\
\hline \multirow[t]{2}{*}{ Query 27} & 0 & 0 & 0 & 13,387 & 16,656 & 0 & 0 & 16,656 \\
\hline & 0.0 & 0.0 & 0.0 & 2.3 & 2.1 & 0.0 & 0.0 & 2.3 \\
\hline \multirow[t]{2}{*}{ Query 28} & 16,656 & 16,656 & $-^{\mathrm{b}}$ & 13,387 & 16,656 & 16,656 & 16,656 & 16,656 \\
\hline & 2.7 & 2.1 & & 2.3 & 2.2 & 2.2 & 2.1 & 2.2 \\
\hline \multirow[t]{2}{*}{ Query 29} & 0 & 0 & 0 & 1,194 & 1,194 & 1,194 & 1,194 & 1,194 \\
\hline & 0.0 & 0.0 & 0.0 & 0.2 & 0.2 & 0.2 & 0.2 & 0.2 \\
\hline \multirow[t]{2}{*}{ Query 30} & 1,194 & 1,194 & $-^{\mathrm{b}}$ & 1,194 & 1,194 & 1,194 & 1,194 & 1,194 \\
\hline & 234.0 & 0.2 & & 0.3 & 0.2 & 0.2 & 0.2 & 0.2 \\
\hline \multirow[t]{2}{*}{ Query 31} & 25 & 25 & 0 & 0 & 11 & 47 & 47 & 47 \\
\hline & 0.0 & 0.0 & 0.0 & 0.0 & 0.0 & 0.0 & 0.0 & 0.0 \\
\hline \multirow[t]{2}{*}{ Query 32} & 43,623 & 43,623 & 0 & 0 & $-\mathrm{b}$ & $-^{\mathrm{b}}$ & $-^{\mathrm{b}}$ & $-^{\mathrm{b}}$ \\
\hline & 5.6 & 5.0 & 0.1 & 0.0 & & & & \\
\hline \multirow[t]{2}{*}{ Query 33} & 6,303 & 6,303 & 6,303 & 31,185 & 31,235 & 31,235 & 31,235 & 31,235 \\
\hline & 0.5 & 0.4 & 5.9 & 3.3 & 10.7 & 10.6 & 11.3 & 11.2 \\
\hline \multirow[t]{2}{*}{ Query 34} & 32 & 32 & 0 & 0 & 34 & 40 & 40 & 40 \\
\hline & 0.0 & 0.0 & 0.0 & 0.0 & 0.0 & 0.0 & 0.0 & 0.0 \\
\hline \multirow[t]{2}{*}{ Query 35} & 1,835 & 1,835 & 0 & 0 & $-^{\mathrm{b}}$ & $-^{\mathrm{b}}$ & $-^{\mathrm{b}}$ & $-^{\mathrm{b}}$ \\
\hline & 0.3 & 0.2 & 0.0 & 0.0 & & & & \\
\hline \multirow[t]{2}{*}{ Query 36} & 0 & 0 & 0 & 960 & 960 & 960 & 960 & 960 \\
\hline & 0.0 & 0.0 & 0.0 & 6.6 & 0.3 & 0.2 & 0.6 & 0.2 \\
\hline \multirow[t]{2}{*}{ Query 37} & 0 & 0 & 0 & 417 & 417 & 417 & 420 & 420 \\
\hline & 0.0 & 0.0 & 0.0 & 0.0 & 0.1 & 0.0 & 11.5 & 11.2 \\
\hline \multirow[t]{2}{*}{ Query 38} & $458^{d}$ & $458^{d}$ & 0 & 0 & $428^{e}$ & $427^{e}$ & $431^{\mathrm{e}}$ & $426^{e}$ \\
\hline & 0.0 & 0.0 & 0.0 & 0.0 & 0.0 & 0.0 & 0.0 & 0.0 \\
\hline \multirow[t]{2}{*}{ Query 39} & 639 & 639 & 0 & 0 & 639 & 639 & 639 & 639 \\
\hline & 0.1 & 0.1 & 0.0 & 0.0 & 0.1 & 0.1 & 0.1 & 0.1 \\
\hline \multirow[t]{2}{*}{ Query 40} & 730 & 730 & 0 & 0 & 737 & 737 & 737 & 737 \\
\hline & 0.1 & 0.1 & 0.0 & 0.0 & 0.1 & 0.1 & 0.1 & 0.1 \\
\hline \multirow[t]{2}{*}{ Query 41} & 5 & 5 & 0 & 9 & 10 & 8 & 9 & 11 \\
\hline & 0.0 & 0.0 & 0.0 & 0.0 & 28.2 & 0.8 & 96.0 & 1.4 \\
\hline \multirow[t]{2}{*}{ Query 42} & 63 & 63 & 0 & 68 & 63 & 63 & 63 & 63 \\
\hline & 0.0 & 0.0 & 0.0 & 0.1 & 0.0 & 0.0 & 0.0 & 0.0 \\
\hline
\end{tabular}

The number of results returned by the system for the given query (in bold), and below, the time in seconds.

b Timeout: System does not return within 5 minutes.

${ }^{\mathrm{c}}$ Error message: XML Parsing Error: not well-formed. Probable cause: query selects GEOMETRY datatype values.

d 36 of the results are different blank nodes.

e Examining the results reveals that result contains duplicates; there are 425 distinct results. 
same number of results or no results, we focus our attention on the queries that do not return results for some systems.

Of the 21 domain queries only 5 return results when issued to Ontop. This is largely because the evaluated version of Ontop does not support the full SPARQL 1.1 query language [65], but only most parts of SPARQL 1.0. Specifically, aggregate functions like MIN, MAX, SUM and COUNT from SPARQL 1.1 are not supported. Also, the well-known workaround of using OPTIONAL and the bound function, which are defined in SPARQL 1.0 and can be used to mimic some of the aggregate functions, are not supported by Ontop. This affects Query 2 (MAX), Query 4 (MIN), Query 6 (COUNT), Query 9 (SUM), Query 10 (SUM) and Query 11 (SUM). The questions behind Query 2 and Query 4 require the use of MAX and MIN since they ask for the latest and earliest time of specific events. Query 6 explicitly asks for the number of wells in a licence, and answering the queries 9,10 and $11^{15}$ requires adding production numbers, in both cases the use of COUNT and SUM, respectively, is unavoidable.

Query 14 uses the SPARQL 1.1 constructs BIND and IF to find the stratigraphic units a wellbore core sample overlaps using the top and bottom depths of the units and the sample. The same question is possible to represent with a slightly different SPARQL query that does not use BIND and IF, essentially by rewriting IF into a different Boolean test using logical-and and logicalor. Hence, even though Ontop does not return any results from Query 14, it is possible to represent the question in the query language fragment that Ontop does support.

Query 15 includes a regex function in a FILTER. This is not supported by the evaluated version of Ontop. The regular expression is used as a straight-forward way of selecting all companies with a name starting with "Statoil". The company commonly known as simply Statoil has had several formal names through history, due to mergers and restructuring, so selecting all previous "versions" of the company is interesting for queries about historic data. All variants of, e.g., Statoil, could of course be selected by a query by explicitly giving all identifiers, but in order to declaratively select all historic instances of a company one would need to transitively follow a relation which relates the old and new company in a so called change-of-name transfer. The current ontology does not support such a query.

Queries 1 and 8 return the error message that the query generated zero rules; we assume that this error is caused by the fact that these queries contain a subquery which use a variable bound by the outer query, since an error message given by earlier versions of Ontop and Fuseki reported this as the cause of the malfunction.

As for Query 16, which asks for all fields with wellbores that are drilled earlier than 2000, for which there is a core sample available, and where the oldest penetrated age is Jurassic, we believe that the reason for zero results returned is that the query contains an individual, the geological era Jurassic. This individual exists only in an external ontology packaged with the OWL ontology that is loaded into Ontop, i.e., it does not exist at the data sources. The functionality of also considering individuals

\footnotetext{
${ }^{15}$ Note that Query 11 is a generalisation of Query 10.
}

given in the ontology, i.e., materialised data, together with the data produced by the mappings, i.e., the virtual ABox, is called hybrid Abox functionality and was first added in the current version of Ontop, and it is a feature that Ontop as the only virtual ABox rewriting system supports. ${ }^{16}$ To test our hypothesis we created a slightly modified version of the query, Query 17, which answers the same question as above, but without the requirement that the oldest penetrated age is Jurassic. Ontop returns 420 results for this query, the same as of the other systems, which supports our assumption.

The domain question that produced Query 12 asks for the kinds of facilities that are used for a field, e.g., oil producing facility. The corresponding query contains rdfs: subClass0f, used to get all subclasses of Facility, and hence requires the reasoner to infer facts from both the TBox and the ABox in order to correctly answer the query. TBox reasoning is generally outside the scope of OBDA systems as the primary goal of OBDA is querying over large datasets. The corresponding query contains a triple ?x rdfs: subClass0f :Facility, used to get all subclasses of Facility, which requires instantiating a variable with a class resource, and querying the type hierarchy. Such queries that combine the type level and instance level of the ontology are generally not supported by OBDA systems. We see that the only systems that return results in our evaluation setup is Fuseki and Stardog without reasoning, returning 1,106 results. Ontop returns zero results, and Stardog with any reasoner enabled does not return results within 5 minutes.

The last queries of the domain queries that do not return results from one or more of the systems are 19, 20 and 21. Query 20 asks for all wellbores drilled before 2000 for which there exist biostratigraphic samples from a cuttings sample, and the log curve is stored online. This query selects data from potentially all data sources, and uses vocabulary that is generated from all three datasets; classes and relations for biostratigraphic samples are defined from the NORLEX dataset, log curves come from the Diskos dataset, and the NPD FactPages vocabulary is natural to use when possible since it plays the role of an upper domain ontology in our setup - and it contains the entry year of wellbores. The query relies on equality reasoning to return answers. A wellbore that occurs in multiple datasets will not have the same URI, all data records from the sources are put in the namespace constructed for each dataset. To identity URIs which represent the same actual wellbore, we use owl: sameAs whenever this information is available in the datasets. Since the query requires reasoning to compute answers, it is no surprise that Fuseki and Stardog without reasoning return no results, and since the OWL 2 QL profile does not permit owl: sameAs statements, this explains why Ontop and Stardog with QL reasoning enabled do not return any results either. However, the OWL profiles EL and RL do support owl: sameAs statements, but unfortunately Stardog does not support this feature: "Only explicit owl: sameAs and owl: differentFrom data assertions will be taken into account for query answering." [66] In order to construct a query that returns results for the question, we need to explicitly encode the

\footnotetext{
${ }^{16}$ http: //ontop-obda.blogspot.it/2013/09/

hybrid-rdf-graphs-or-hybrid-aboxes-as.html
} 
equality relationship into the query, this is done in Query 21. By interpreting the query in a literal manner, we see that it asks for three different wellbores from each of the datasets, collecting different information about them from each set, and requires that the three wellbores are related by the owl: sameAs relation. Note that the direction of the relation is not arbitrary; since Stardog and Fuseki (in our evaluation setup) do not infer any new facts from owl: sameAs statements, e.g., that it is a symmetric relation, the query can only make use of facts that are explicit in the dataset. For Query 21 all configurations of Stardog return 68 results, Fuseki times out, and Ontop returns zero results.

Unit test queries. The unit test queries are designed to test specific features of the query answering capabilities of the systems, by using small and focused queries. However, since some test functionality of one particular system might not be supported by other systems, some of these tests give unexpected results, which might well be due to a malformed query from the viewpoint of the system rather then ill-behaviour by the system. We discuss them in turn, although identifying the cause of all discrepancies between query answers is outside the scope of this paper.

Query 22 selects all resources that are related by owl: sameAs. As expected, Fuseki and Stardog return the same numbers of results, 7,441, confirming that Stardog does not perform any equality reasoning. Ontop, however, returns 7,312 results, which is roughly the set of answers returned from the other systems, but without the owl: sameAs relationship that exists in the ontology files.

Query 23 effectively asks for wellbores that have the same name in the NPD FactPages dataset and the NORLEX database, requiring that $n l x v$ : Wellbores are inferred as $n p d v$ : Wellbores using a subclass axiom. All systems return the expected results.

Queries 24, 25 and 26 ask for geographical data. Running queries 24 and 25 against Ontop gives a parsing error, which we believe is caused by the mapping of the GEOMETRY datatype. Ontop did not accept a mapping were the geographical data was converted into text; the data is therefore probably given to the endpoint in an unexpected format, which results in the error. By removing the geographical values from the query, giving Query 26, Ontop returned the expected answers, although without removing duplicates.

With Query 27 and Query 28 we test the support of inverse object properties. The property npdv: isGeometry0fFeature is defined as the inverse property of geo:hasGeometry. Inverse properties are included in the OWL profiles QL and RL and as expected only Ontop and Stardog with QL or RL reasoning enabled returned any answers. By formulating the query using the SPARQL inverse path construction, we were able to return the same results as for Query 27, but for all systems (excluding D2RQ). This shows that it is possible in some cases to use query constructs as substitutes for lack of reasoning support. There were less answers returned by Ontop for these queries; we assume this is due to the different problems related to the representation of geographical data using Ontop. Query 29 and Query 30 show similar results; the first uses alternate property paths and gives the same results as the latter query, which uses the super property of the alternate properties of the first.
The queries 31, 32 and 33; and 34, 35 and 36 are two groups of queries that respectively ask for all subclasses of a given class, all instances of subclasses of the same class, and all instances of the given class. Their results indicate firstly, that queries that require combined type level and instance level reasoning are not supported by rewriting systems as Ontop. Secondly, combined reasoning is computational expensive, and lastly, the simple fact that reasoning can dramatically increase the number of answers for a query.

Query 37 is one of the few of the queries in the evaluation that returns different results of different profiles in Stardog. The query asks for all discovery wellbores. Note that the time dramatically increases for the configurations that return more answers.

The queries 38, 39 and 40 return respectively all instances of owl:Class, rdf:Property, and of owl:ObjectProperty, owl: DatatypeProperty and owl: AnnotationProperty. For the first query, Stardog does not remove duplicate answers although DISTINCT is used in the query. Otherwise, the results are as expected.

Query 41 asks for all the types of a particular wellbore individual. We see that the different profiles give different answers. However, using a more general query, Query 42, all triples where the subject is this same wellbore individual, then Fuseki and Stardog with any reasoning configuration return the same answers, and with less types than in Query 41. Ontop returns a few more results than the other systems. We believe the reason for these results are that Query 42, due to its very general graph pattern, leaves the type of the predicate variable [67, ch. 7.1.3] unspecified. Stardog handles this by computing results from explicit triples only, while Ontop performs regular reasoning.

\section{Conclusion and Future Work}

We observed that the transformation of existing real-world datasets into high-quality semantic web data is a complex task that requires the concerted execution of a variety of processing steps using a range of different tools. Creation and maintenance of such data sets in the face of changing data and requirements leads to a significant engineering challenge, that requires appropriate tool support.

The main contribution of our work is a proposed architecture that ties together and drives all the required components for all configurations used in the transformation and publication process. This architecture hinges on a single declarative description or specification of the whole setup, that is translated into an ontology, mappings, and any other objects required to drive the tool chain. In this way, firstly, any changes to the involved tools, data, schemata, ontology, etc., are limited to single points of change (either in the specification itself or in the translation rules), and secondly, the specification itself becomes an engineering artefact that can be subjected to version control, documentation, tool support, and more.

We have performed a thorough case study where we transform a number of relational (SQL) and tabular (CSV) datasets from the petroleum industry domain into high-quality RDF data, using a variety of approaches and tools. In particular, we included approaches using materialised RDF triple stores, as well as 'virtual' 
stores where incoming SPARQL queries are translated to queries over the data sources in their original form. We also included tools with reasoning support for different fragments of the OWL language.

Using a collection of queries elicited directly from individuals in the petroleum industry who work with the underlying data on a daily basis, we could run a comparative evaluation of the suitability of the different approaches for the chosen queries, using the particular tools chosen, on that particular data. We reported the results in terms of evaluation time and completeness of query answering.

However, the results of that comparison are clearly ephemeral, since tools evolve rapidly, requirements will be different for different data or different users of the same data, etc. We therefore stress the importance of conducting query evaluations using "real" queries when deciding on what OBDA system architecture and tools to deploy.

In future work, we will extend the specification language with more features and develop and release open source tools that implement the specification and that can leverage existing tools in the bootstrapping process of the OBDA specification. We will also adopt the R2RML standard for mappings as part of our architecture, as it starts to be adopted by an increasing number of tools we use. We also want to investigate the possibility of configuring visual query interfaces (like, e.g., [68]) for the produced data sets from the same declarative specifications.

We also intend to continue work on the petroleum domain case study, refining the ontology and possibly integrating further datasets. The results will play an important role for the industrial use cases of the Optique project [69, 70].

\section{Acknowledgements}

This research was partially funded by the the Seventh Framework Program (FP7) of the European Commission under Grant Agreement 318338, "Optique"17 and The Research Council of Norway through the Semicolon II project ${ }^{18}$. We thank the employees of Statoil who provided the domain queries. Thanks to the Norwegian Petroleum Directorate (NPD) for making the data of the NPD FactPages available as open data, to Øyvind Hammer at the Natural History Museum in Oslo for giving us access to the NORLEX database dump, and to Atul Solanki at Halliburton Landmark Software \& Services for providing access to the Diskos database. Also thanks to the anonymous reviewers whose feedback greatly improved the presentation of this article.

\section{A. The OBDA Engineering Specification Language}

The OBDA engineering specification language is a declarative language for describing a set of maps that specify the translation of a common set of modelling structures into a relational

\footnotetext{
${ }^{17}$ http: //www.optique-project.eu/

${ }^{18}$ http://www. semicolon.no/
}

database, an ontology and mappings between the ontology and database. These constructs are (1) entities, (2) identifiers, (3) attributes, (4) relations, and (5) subtypes. How the maps of these constructs are represented in the specification language is listed in Figure A.16. ${ }^{19}$ When referring to a field, for readability, we include the short name of the field, which is unique among the fields for a map. For example, "E1 ( $t b l)$ " refers to the table name field of the entity map.

The specification language description is functional in the sense that we do not prescribe a specific serialisation for representing specification instances. In the use case that has driven the development of the specification language, the OBDA use case specification was represented as a set of CSV files, but other formats, like RDF or XML, could have been used.

The syntactic requirements of the current version of the specification language, and the rules for translating an OBDA specification for a set of source data into a relational database, an ontology, and mappings, are given below. For examples and an illustration of use of the specification, we refer to Sections 2 and 3 .

\section{A.1. Syntactic Requirements}

The syntactic and structural requirements that a specification instance must abide by are given as two lists. The first list presents the permissible values for the different fields in a specification and the second list contains the referential constraints between fields in a specification.

Permissible values. Default values are used when no value is set for a field. A field without value and for which no default value is set, is an unspecified field.

- E1 $(t b l)$ and A1 (col) must contain legal table and column names respectively.

- E5 (URI), A5 (URI), R5 (URI), S3 (URI) must contain legal URIs [71], or no value.

- E2 (lab), E6 (desc), A4 (lab), A11 (desc) can contain arbitrary strings.

- I3 (patn) and A8 (patn) must contain a URI with special role fillers, or no value.

- A6 (type) must contain one of the values D (DataProperty), A (AnnotationProperty), 0 (ObjectProperty), or OP (ObjectPropertyPattern), or no value. The default value is $D$.

- A7 (tran) and S6 (tran) must refer to a transformation table, or contain no value. The default value is the identity function.

- A9 (lang) must contain a legal RDF language tag [72], or no value.

\footnotetext{
${ }^{19}$ This table is a copy of Figure 4 that we included here for easy reference and in order to make this description of the specification language self-contained.
} 


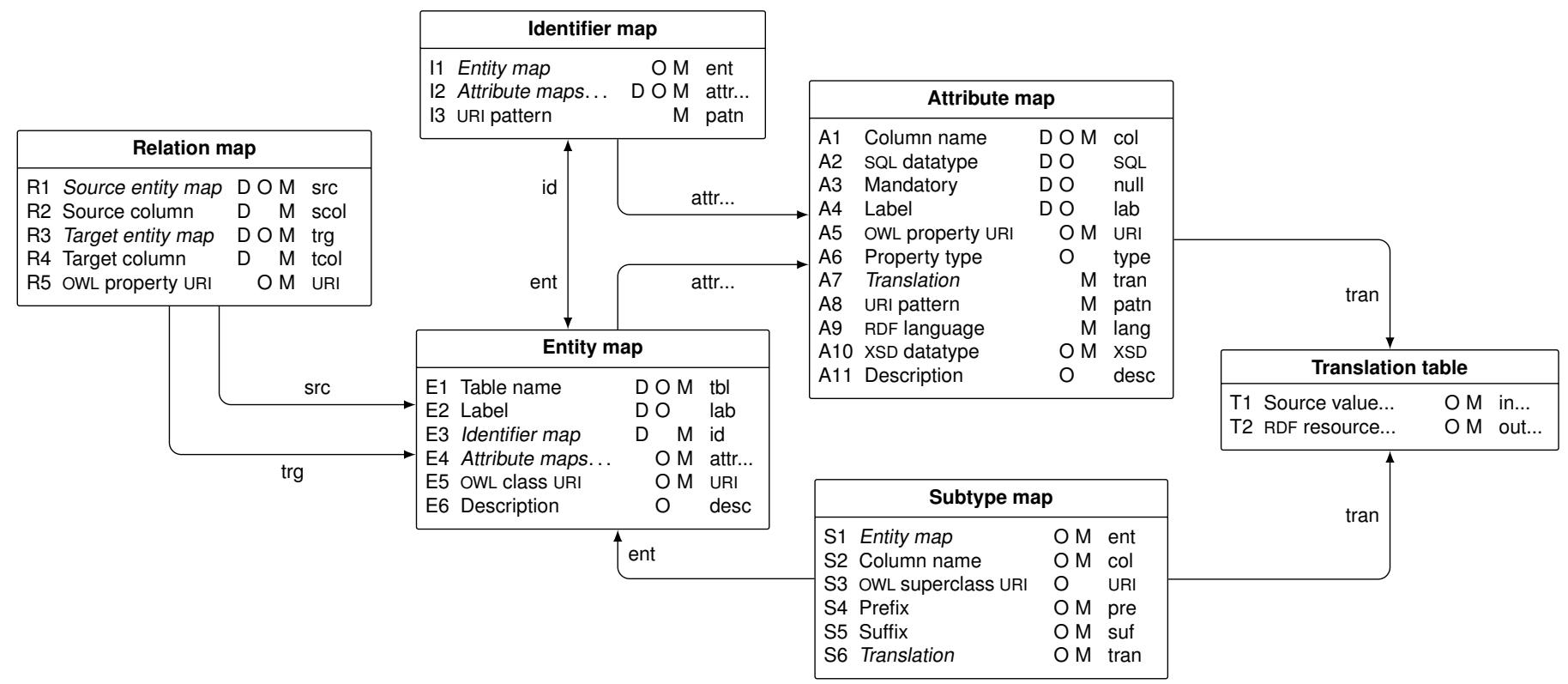

Figure A.16: Schematic overview of the OBDA engineering specification. (This figure is a copy of Figure 4.)

- A10 (XSD) must contain a URI denoting an RDF datatype, or no value.

- A2 (SQL) must contain a string denoting a SQL datatype, or no value.

- A3 (null) must contain a Boolean value.

- S4 (pre) and S5 (suf) must contain strings that can form part of a URI, or no value. The default value is the empty string.

\section{Reference requirements:}

- An entity map refers to an identifier map (E3 (id)) and a list of attribute maps (E4 (attr...)).

- An identifier map refers to an entity map (I1 (ent)) and a list of attribute maps (I2 (attr...)).

- An attribute map can refer to a transformation table (A7 (tran)).

- For a relation map, source and target entity maps, R1 (src) and R3 (trg), refer each to some entity map; and source and target columns, R2 (scol) and R4 (tcol), refer each to some column name (A1 $(\mathrm{col}))$.

- A subtype map refers to an entity map (S1 (ent)) and a column name $(\mathrm{S} 2(\mathrm{col}) \rightarrow \mathrm{A} 1(\mathrm{col}))$.

\section{A.2. Rules}

When referring to specification values, we use the following dot notation. Let $\mathbf{m}$ be a map and V1 the short name of a field (found in Figure A.16) available for this map, then m.V1 denotes the value of this field. ${ }^{20}$ If a value is a reference to a map, then we can "chain" the dot notation, for instance, if $\mathbf{m}$.V1 refers to an entity map, then m.V1.URI denotes the OWL class URI set for this entity map. To indicate a set or list of values, we use three dots: m.V2... We also allow ourselves to chain lists; assuming that V2 is a list of references to other maps, m.V2...V3 is the list of V3-values set for each of the V2 maps of $\mathbf{m}$.

\section{A.2.1. Database Rules}

Database rules specify how an OBDA engineering specification is translated into a relational database.

When defining the rules to construct a relational database from the OBDA engineering specification, we use the following pragmatic definition of a database schema.

A database schema is a set of tables and a set of foreign keys. A table is specified with a table name, a primary key, an optional table comment and a set of columns. A column is specified with a column name-which is unique for the table, an SQL datatype, a mandatory flag, and an optional column comment. A primary key is specified as a list of column names. A foreign key is specified with a source table name, source column name, target table name and target column name.

Database Rule 1 specifies how to construct tables with columns and primary keys. Database Rule 2 constructs foreign keys.

Database Rule 1 (Tables) For each entity map e, create a table by setting

\footnotetext{
${ }^{20}$ For example, if $\mathbf{e}$ is the entity map in Figure 5a line 1, then e.URI is the value npdv: FieldOperator.
} 
- the table name to e.tbl, and

- the table comment to e.lab.

- The primary key is declared as the set of column names e.id.attr...col.

- For every field name $\mathbf{f}$ in the tabular file to which the table name corresponds, let $\mathbf{a}$ be the attribute map where the column name a.col is equal to $\mathbf{f}$ and add a column to the table by setting

- the column name to a.col,

- the column datatype to a. $S Q L$,

- the column mandatory flag to a.null, and

- the column comment to a.lab.

Note that Database Rule 1 uses not only the specification, but reads column names out of the first row of the tabular files to be represented in a database.

Database Rule 2 (Foreign keys) For each relation map $\mathbf{r}$, create a foreign key by setting

- the source table to r.src.tbl,

- the source column to r.scol,

- the target table to r.trg.tbl, and

- the source column to r.tcol.

\section{A.2.2. Ontology Rules}

Ontology rules specify how an OBDA engineering specification is translated into a ontology axioms. In order to keep the rules simple and free from too may exceptions, we assume that an axiom that involves a specification field that is unspecified is not created.

Ontology Rule 1 declares classes, Ontology Rule 2 declares properties, and Ontology Rule 3 constructs keys. Ontology Rule 4 and Ontology Rule 5 construct axioms that reflect respectively mandatory attributes and foreign keys. Ontology Rule 6 extracts subclasses from the source data.

Ontology Rule 1 (Classes) For each entity map e, create the following axioms:

\section{Declaration(Class(e.URI))}

AnnotationAssertion(rdfs:label e.URI e.lab)

AnnotationAssertion( $r d f s$ : comment e.URI e.desc)

Ontology Rule 2 (Properties) For each attribute map a, create the following axioms:

$\operatorname{Declaration}(X(\mathbf{a} . U R I))$

AnnotationAssertion(rdfs:label a.URI a.lab)

AnnotationAssertion(rdfs : comment a.URI a.desc)

where $X$ has the following value:

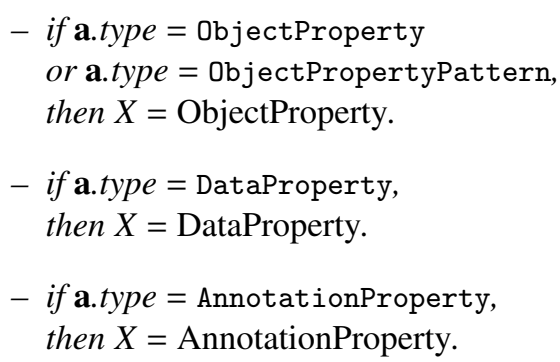

Ontology Rule 3 (Keys) For each identifier map i, if every $\mathbf{a}$ in i.attr... occurs in i.ent.attr..., create the following axiom:

$$
\left.\operatorname{HasKey(i.ent.URI}\left(\mathbf{a}_{o} \ldots U R I\right)\left(\mathbf{a}_{d} \ldots U R I\right)\right)
$$

where $\mathbf{a}_{o} \ldots$ is the list of attribute maps in $\mathbf{i}$.attr... that specify an object property, and $\mathbf{a}_{p} \ldots$ is the list of attribute maps in i.attr... that specify a datatype property.

Ontology Rule 4 (Mandatory properties) For each entity map $\mathbf{e}$, and for each attribute map $\mathbf{a}$ in $\mathbf{e}$.attr..., if $\mathbf{a}$ maps to an object property or a datatype property, and $\mathbf{a}$.null is true, create the following axiom:

\section{SubClassOf(e.URI A(a.URI X))}

where $A$ and $X$ have the following values:

- if a maps to an object property, then $A$ is ObjectSomeValuesFrom and $X$ is owl: Thing,

- if a maps to a datatype property, then $A$ is DataSomeValuesFrom and $X$ is rdfs : Literal.

Ontology Rule 5 (Relation) For each relation map $\mathbf{r}$, create the following axioms:

\section{Declaration(ObjectProperty(r.URI)) \\ SubClassOf(r.src.URI \\ ObjectSomeValuesFrom(r.URI \\ r.trg.URI))}

Unlike the rules presented so far, the next rule also directly handles values collected from the source data. We give some preliminary definitions to address the translation of these values.

A translation table is a partial function that maps strings (of source data) to strings (representing RDF resources). If the function is not defined for an input string, the output is unspecifiedand the affected axiom is hence not created.

Let URISubtype $(\mathbf{s}, v)$ be a function that takes a subtype map $\mathbf{S}$ and a string $v$ as input, and outputs a string that is the concatenation of s.pre, s.tran(v), s.suf. The function transforms the input value with the translation table in s.tran, and prefixes and suffixes it with s.pre and s.suf, respectively.

Ontology Rule 6 (Subclasses from data) For each subtype map $\mathbf{s}$, let $C$ be the set of answers from the following query over the source data: 
SELECT s.col FROM s.ent.tbl.

For each $c \in C$, add the following axioms:

Declaration(Class(URIsubtype(s c $)$ ))

SubClassOf(URIsubtype(s $c$ ) $\mathbf{s . U R I )}$

\section{A.2.3. Mapping Rules}

Mapping rules specify how an OBDA engineering specification is translated into mappings that relate the ontology to the source data on relational format.

The mappings are described by two parts. The first is an SQL query over the source database, the second an RDF triple pattern in turtle serialisation which may contain "role filler" parts placed in \{curly braces\} that refer to names in the SELECT clause of the SQL query, using the same format as for a template-valued term map in the R2RML specification [37].

To help build such patterns we introduce the function URIpat(pattern, cols...) that takes as input a URI pattern of the form used in the OBDA specification, where role filler parts are indicated with strings of the form " $\{\$ 1\}$ ", and a list of column names cols.... The function returns a URI pattern on the R2RML format by replacing each role filler in the input pattern consecutively with the input column names surrounded with curly braces; for example, URIpat(http: //example.org/ $\{\$ 1\} / \mathrm{bbb} /\{\$ 2\} / \mathrm{ddd}$, aaa, ccc) returns http://example.org/ $\{$ aaa $\} /$ bbb/ $\{c c c\} / d d d$. Let URIentpat be the function that returns the URIpattern for an entity map: URIentpat(e) = URIpat(e.id.patn, e.id.attr...col).

The function SQL2XSD maps a standard set of SQL datatypes to XSD datatypes, for instance SQL2XSD(INT) = xsd: integer.

Mapping Rule 1 specifies mappings to create individuals, while Mapping Rule 2 and Mapping Rule 3 make mappings that create relationships between individuals. Mapping Rule 4 results in mappings that produce the membership statements to those subclasses extracted from the source data by Ontology Rule 6.

Mapping Rule 1 (Individuals) For each entity map e, create the following mapping:

DB: SELECT e.id.attr...col FROM e.tbl

$R D F:<U R I$ entpat(e)> rdf : type <e.URI>

Mapping Rule 2 (Relationships) For each entity map e, and for each $\mathbf{a}$ in e.attr..., create the following mapping:

DB: SELECT e.id.attr...col, a.col FROM e.tbl

$R D F:\langle U R I e n t p a t(\mathbf{e})><\mathbf{a} . U R I>O$

where O has the following value:

- if a maps to a datatype property and

- if $\mathbf{a} . X S D$ is specified, then $O="\{\mathbf{a} . c o l\} "$ "^a. $X S D$;

- if SQL2XSD(a. $S Q L)$ is specified, then $O=$ "\{a.col $\} "$ "^SQL2XSD(a.SQL);

- if $\mathbf{a}$.lang is specified, then $O=$ "\{a.col $\} " @ \mathbf{a}$.lang;
- if a maps to an object property and

- if a.type is ObjectPropertyPattern, then $O=\langle U R I p a t(\mathbf{a}$. patn, a.col) $\rangle$;

- if a.type is ObjectProperty, then $O=\langle\{$ a.tran $(\mathbf{a}$. col $)\}\rangle$;

- else $O="\{\mathbf{a} . \mathrm{col}\} "$.

Mapping Rule 3 (Relations) For each relation map $\mathbf{r}$, create the following mapping:

DB: SELECT r.src.id.attr...col, r.trg.id.attr...col FROM r.src.tbl, r.trg.tbl

$R D F:\langle U R I$ entpat(r. $s r c)\rangle\langle\mathbf{r}$.URI $\rangle\langle$ URIentpat(r.trg) $\rangle$

Mapping Rule 4 (Subclasses from data) For each subtype map $\mathbf{s}$, let $C$ be the set of answers from the following query over the source data:

SELECT s.col FROM s.ent.tbl.

For each $c \in C$, add the following mapping:

DB: SELECT s.ent.id.attr...col, s.col FROM S.ent.tbl

RDF: <URIentpat(s.ent)> rdf : type <URISubtype(s, $c)\rangle$

\section{B. Queries}

All queries used in the use case query experiment are listed here, partitioned in two: domain expert queries, which are queries derived from real questions from domain experts, and unit test queries, which are aimed to complement the domain expert queries for the testing of the OBDA systems. The domain expert queries are also given with the original question.

\section{B.1. Domain Expert Queries}

Query 1. Who are the licensees of production licence $X$ and how big is their share?

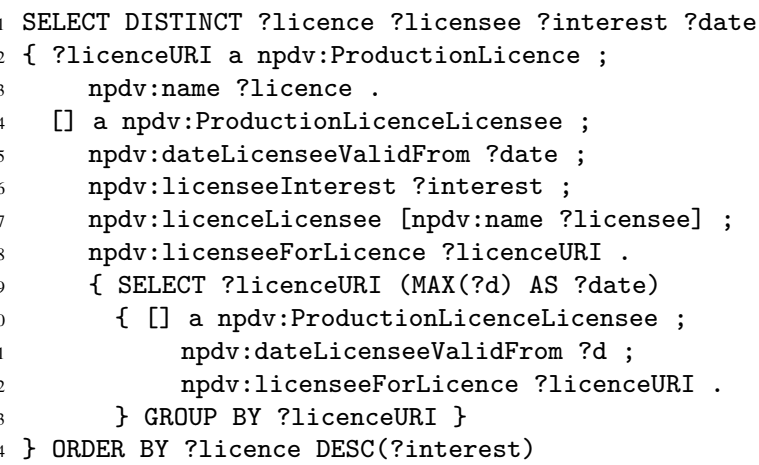


Query 2. Who is the operator of licence X?

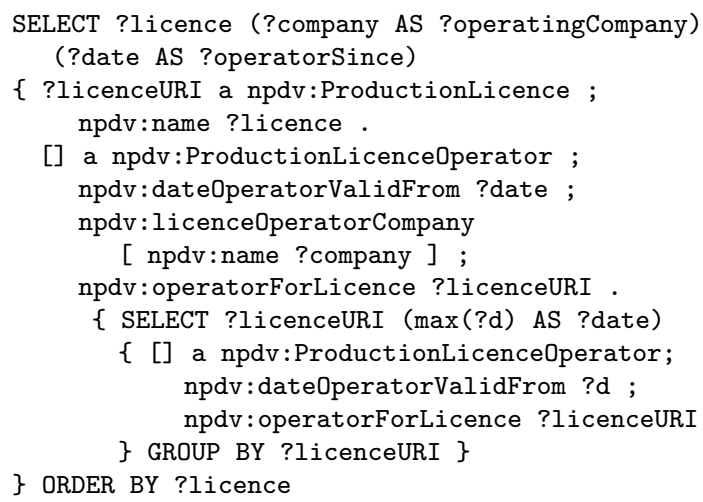

Query 3. When was the licence $X$ granted and to when is it valid?

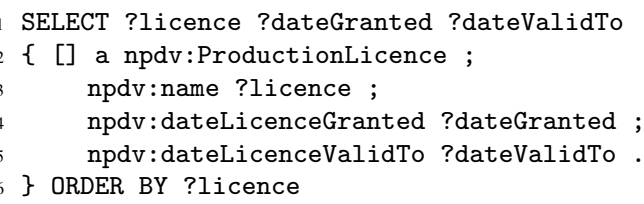

Query 4. When was the first well drilled in licence X?

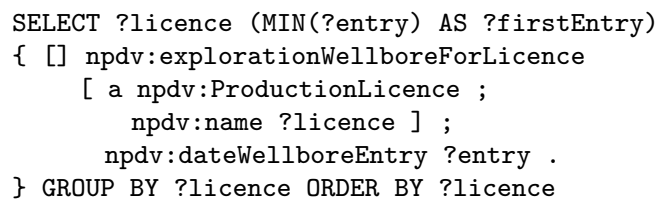

Query 5. What companies have been owners in licence X?

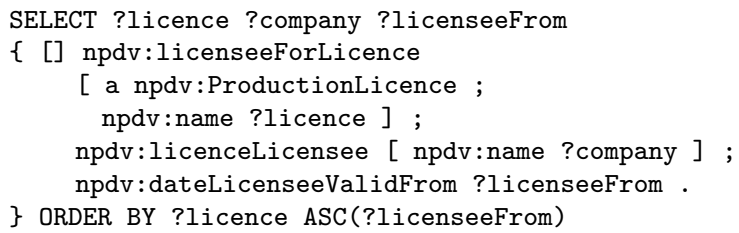

\section{Query 6. How many wells have been drilled in licence $X$ ?}

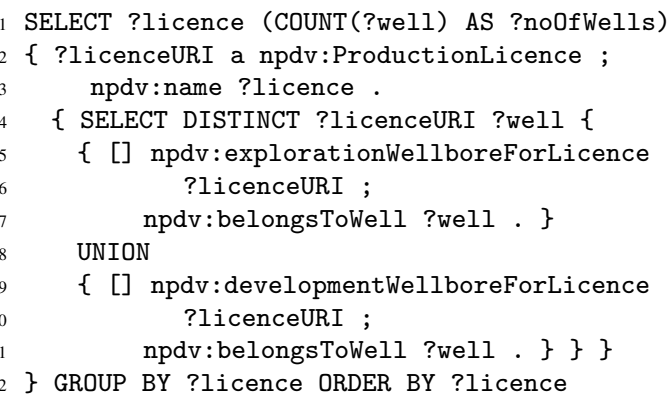

Query 7. What is the estimated reserves for field $X$ and how much oil, gas and condensate does the reserves consist of?

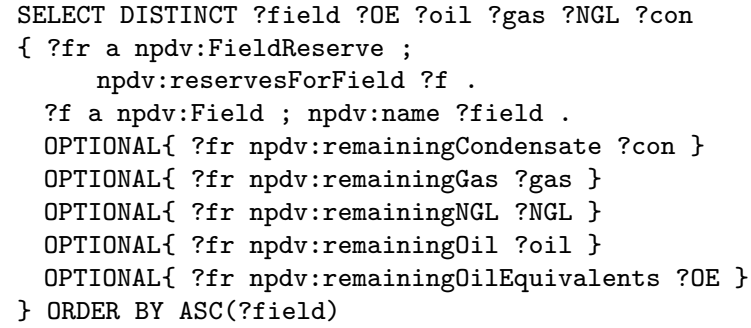

\section{Query 8. What is/was the top production month for field X?}

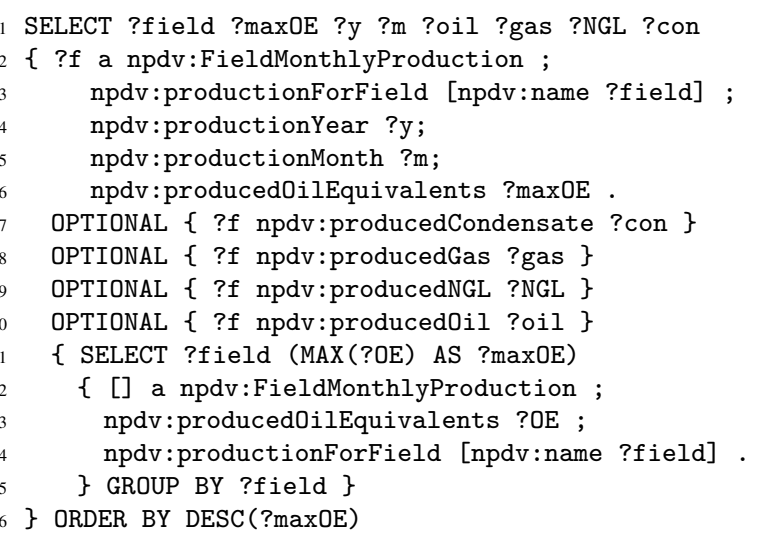

Query 9. What is the total production of field X?

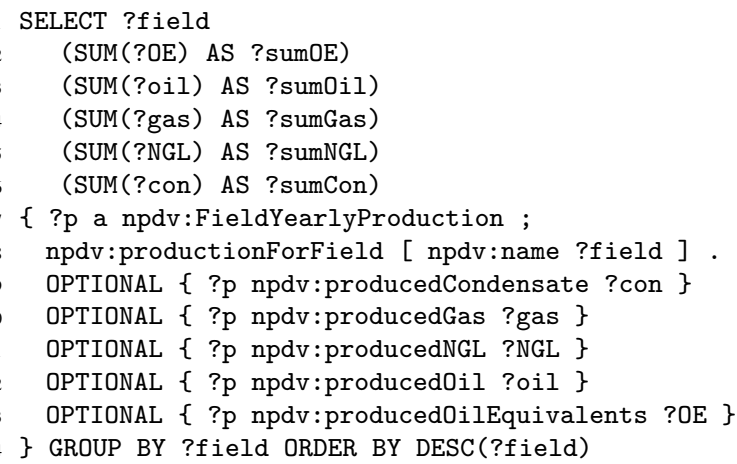

Query 10. What was the total production of oil and gas in the period Jan-Jun 2010 for fields where Statoil was operator?

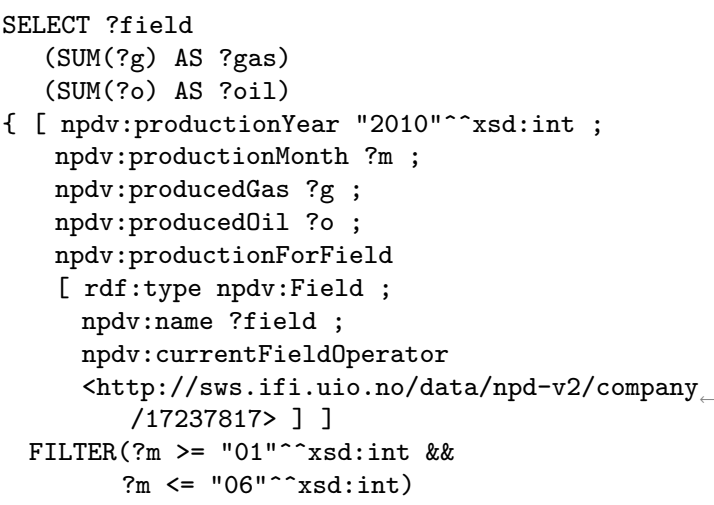


Query 11.

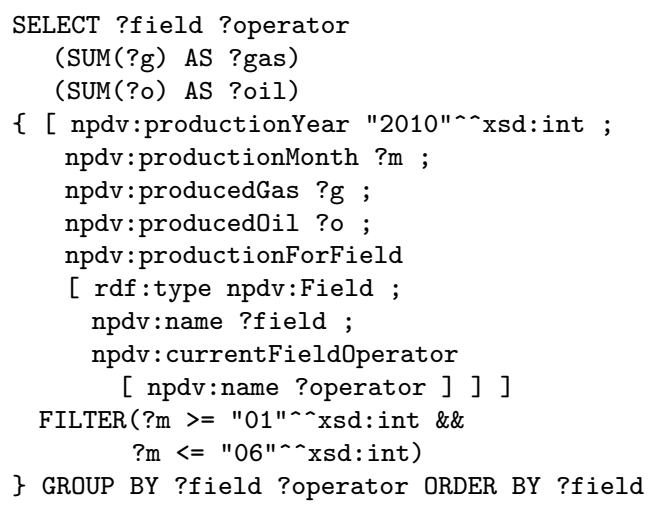

Query 12. What kind of facility is used for field X?

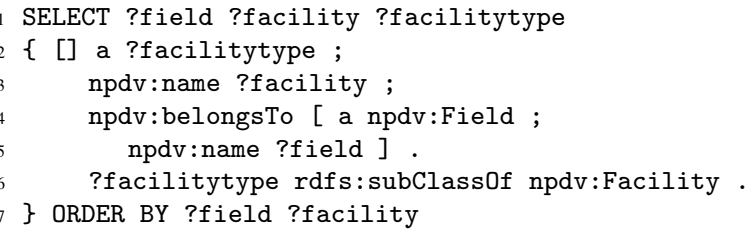

Query 13. What are the wellbores with total core length greater than $30 \mathrm{~m}$ ?

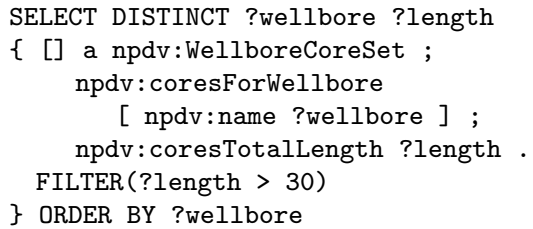

Query 14. What wellbores have cores in a specific set of stratigraphic units?

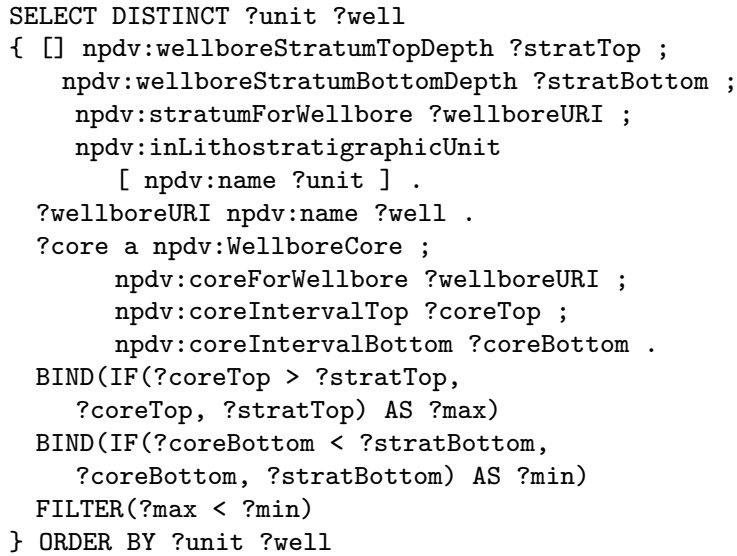

Query 15. List wellbores completed before 2008 where Statoil as drilling operator sampled less than $50 \mathrm{~m}$ of core $(\mathrm{s})$.

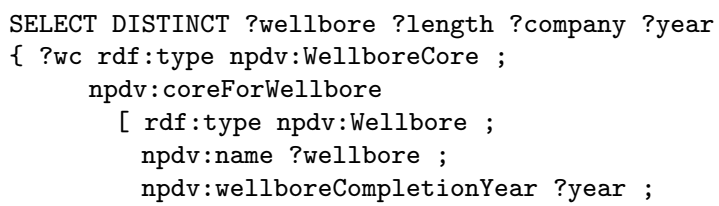

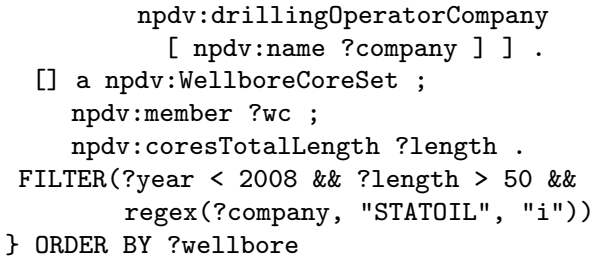

Query 16. What fields have wellbores that are drilled earlier than 2000, for which there is a core sample available, and where the oldest penetrated age is Jurassic?

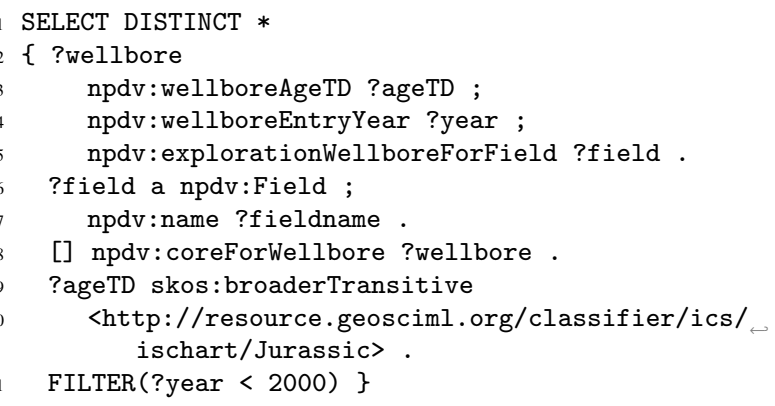

Query 17. What fields have wellbores that are drilled earlier than 2000, and for which there is a core sample available? This is a slightly modified version version of Query 16 used to test whether Ontop returns answers when the use of its hybrid Abox functionality is not necessary.

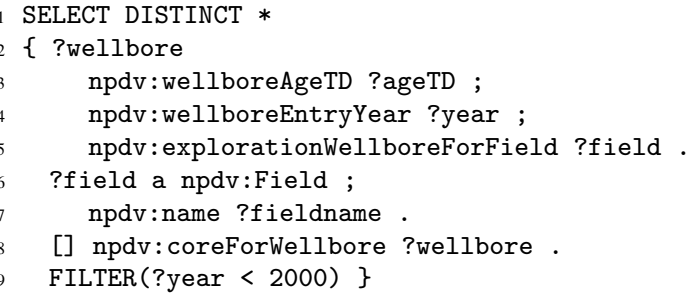

Query 19. List the fields and wellbores with their operator for which there exists a electromagnetic log curve.

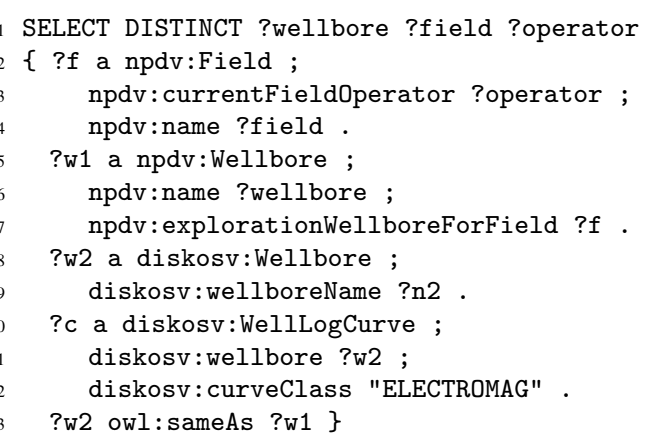

Query 20. List wellbores drilled before 2000 for which there exist biostratigraphic samples from a cuttings sample, and the log curve is stored online.

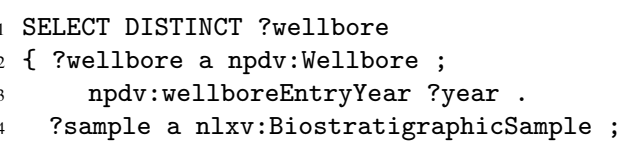




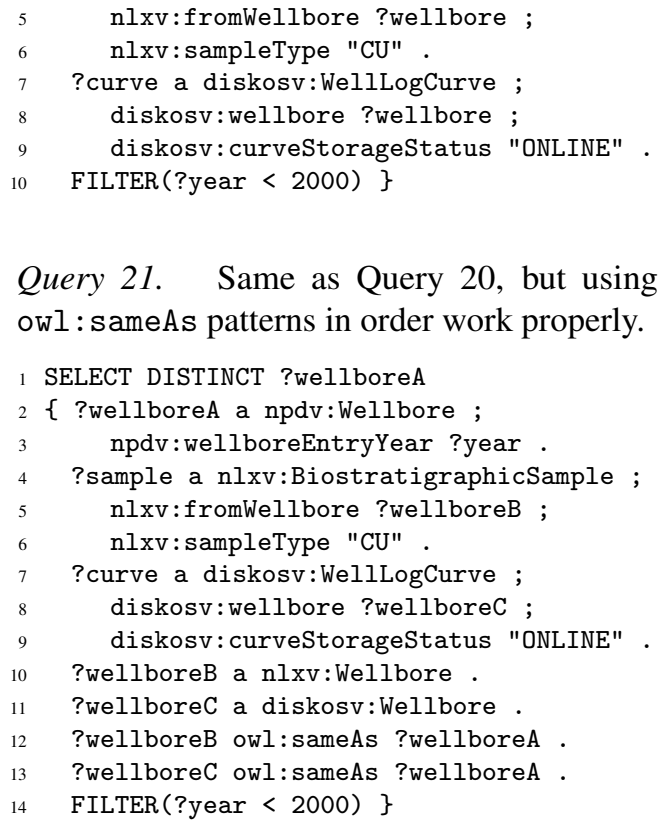

\section{B.2. Unit Test Queries}

Query 22.

1 SELECT DISTINCT $*\{$ ? $\mathrm{x}$ owl:sameAs ?y $\}$

\section{Query 23.}

1 SELECT DISTINCT ?n

$2\{$ ?x a npdv:Wellbore ; nlxv:wellboreName ?n.

3 ?y a npdv:Wellbore; npdv:name ?n \}

\section{Query 24.}

1 SELECT DISTINCT ?feature ?geometry ?WKT

2 \{ ?geometry geos:asWKT ?WKT ;

npdv:isGeometryOfFeature ?feature \}

\section{Query 25.}

SELECT DISTINCT ?feature ?geometry ?WKT

$2\{$ ?geometry a geos:Spatialobject, npdv:Point ; geos:asWKT ?WKT ;

npdv:isGeometryOfFeature ?feature \}

\section{Query 26.}

\section{SELECT DISTINCT ?feature ?geometry}

$2\{$ ?geometry a geos:SpatialObject, npdv:Point ; npdv: isGeometry0fFeature ?feature

\section{Query 27.}

SELECT DISTINCT ?feature ?geometry

$2\{$ ?feature geos:hasGeometry ?geometry \}

\section{Query 28.}

1 SELECT DISTINCT ?feature ?geometry

$2\{$ ?feature npdv:isGeometryOfFeature ?geometry \}
Query 29.

SELECT DISTINCT *

$\{$ ?s npdv: wellboreAgeHcLevel1

npdv: wellboreAgeHcLevel2

npdv: wellboreAgeHcLevel3 ?o \}

Query 30.

1 SELECT DISTINCT * $\{$ ?s npdv: wellboreAgeHc ?o $\}$

Query 31.

1 SELECT DISTINCT ?class

2 ? class rdfs:subClassOf npdv:Wellbore \}

Query 32.

1 SELECT DISTINCT ?class ?instance

$2\{$ ?class rdfs:subClass0f npdv:Wellbore.

3 ?instance rdf:type ?class \}

Query 33.

1 SELECT DISTINCT ?instance

$2\{$ ?instance rdf:type npdv:Wellbore $\}$

Query 34.

1 SELECT DISTINCT ?class

$2\{$ ?class rdfs:subClass0f npdv:Facility.

3 \} ORDER BY ?class

Query 35.

1 SELECT DISTINCT ?class ?facility

$2\{$ ?class rdfs:subClass0f npdv:Facility.

[] rdf:type ?class ; npdv:name ?facility .

5 \} ORDER BY ?facility

Query 36.

SELECT DISTINCT ?facility

\{ [] a npdv:Facility ;

npdv:name ?facility

4 \} ORDER BY ?facility

Query 37.

1 SELECT DISTINCT ?wellbore

$2\{$ ?wellbore a npdv:DiscoveryWellbore \}

Query 38.

1 SELECT DISTINCT ?s \{ ?s a owl:Class \}

Query 39.

1 SELECT DISTINCT ?s \{ ?s a rdf:Property $\}$

Query 40.

1 SELECT DISTINCT ?s

$2\{\{$ ?s a owl:ObjectProperty $\}$ UNION

\{ ?s a owl:DatatypeProperty $\}$ UNION

\{ ?s a owl:AnnotationProperty $\}$ \} 
Query 41.

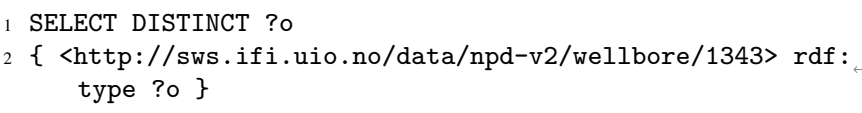

\section{Query 42.}

1 SELECT DISTINCT ?p ?o

$2\{$ <http://sws.ifi.uio.no/data/npd-v2/wellbore/1343> ?p ? o

3 \} ORDER by ?p

\section{References}

1. Free Software Foundation. GNU Make. Online; Accessed June 2014. 2014. URL: https : //www.gnu.org/software/ make/.

2. The Apache Software Foundation. Apache Ant. Online; Accessed June 2014. 2014. URL: https : / / ant . apache . org/.

3. The Apache Software Foundation. Apache Maven. Online; Accessed June 2014. 2014. URL: https : //maven . apache . org/.

4. Richard Cyganiak, David Wood, and Markus Lanthaler, eds. RDF 1.1 Concepts and Abstract Syntax. W3C Recommendation. Feb. 2014. URL: http://www . w3.org/TR/rdfconcepts/.

5. The W3C SPARQL Working Group. SPARQL 1.1 Overview. W3C Recommendation. W3C, 2013. uRL: http: //www .w3.org/TR/sparql11-overview/.

6. Christian Bizer, Tom Heath, and Tim Berners-Lee. "Linked Data - The Story So Far". In: International Journal on Semantic Web and Information Systems 5.3 (2009), pp. 1-22. Dor: 10.4018/jswis. 2009081901.

7. Yuanbo Guo, Zhengxiang Pan, and Jeff Heflin. “An Evaluation of Knowledge Base Systems for Large OWL Datasets". In: International Semantic Web Conference. Ed. by Sheila A. McIlraith, Dimitris Plexousakis, and Frank van Harmelen. Vol. 3298. Lecture Notes in Computer Science. Springer, 2004, pp. 274-288. URL: http://dblp. unitrier.de/db/conf/semweb/iswc2004.html\#GuoPH04.

8. Christian Bizer and Andreas Schultz. "The Berlin SPARQL Benchmark”. In: Int. J. Semantic Web Inf. Syst. 5.2 (2009), pp. 1-24.

9. Mariano Rodriguez-Muro, Roman Kontchakov, and Michael Zakharyaschev. "Ontology-Based Data Access: Ontop of Databases". In: The Semantic Web - ISWC 2013. Ed. by Harith Alani, Lalana Kagal, Achille Fokoue, Paul Groth, Chris Biemann, Josiane Xavier Parreira, Lora Aroyo, Natasha Noy, Chris Welty, and Krzysztof Janowicz. Vol. 8218. Lecture Notes in Computer Science. Springer Berlin Heidelberg, 2013, pp. 558-573. Dor: 10.1007/9783-642-41335-3_35. URL: http://dx.doi .org/10 . 1007/ 978-3-642-41335-3_35.
10. Juan Sequeda and Daniel P. Miranker. "Ultrawrap: SPARQL execution on relational data". In: J. Web Sem. 22 (2013), pp. 19-39.

11. Orri Erling and Ivan Mikhailov. "RDF Support in the Virtuoso DBMS”. In: Networked Knowledge-Networked Media. Springer, 2009, pp. 7-24.

12. Davide Lanti, Martin Rezk, Mindaugas Slusnys, Guohui Xiao, and Diego Calvanese. "The NPD Benchmark for OBDA Systems". In: Proc. of the 10th Int. Workshop on Scalable Semantic Web Knowledge Base Systems (SSWS 2014). Vol. 1261. CEUR Electronic Workshop Proceedings, http://ceur-ws.org/. 2014, pp. 3-18.

13. Juan F. Sequeda, Marcelo Arenas, and Daniel P. Miranker. "OBDA: Query Rewriting or Materialization? In Practice, Both!" In: The Semantic Web - ISWC 2014 - 13th International Semantic Web Conference, Riva del Garda, Italy, October 19-23, 2014. Proceedings, Part I. 2014, pp. 535551. DOI: 10 . $1007 / 978-3-319-11964-9 \_34$. URL: http : //dx.doi.org/10.1007/978-3-319-11964-9_34.

14. Boris Motik, Peter F. Patel-Schneider, and Bijan Parsia, eds. OWL 2 Web Ontology Language Structural Specification and Functional-Style Syntax (Second edition). W3C Recommendation. Dec. 2012. URL: http: //www .w3.org/ TR/ow12-syntax/.

15. Ralf Möller, Volker Haarslev, and Michael Wessel. "On the Scalability of Description Logic Instance Retrieval”. In: 29. Deutsche Jahrestagung für Künstliche Intelligenz. Ed. by C. Freksa and M. Kohlhase. Lecture Notes in Artificial Intelligence. Springer, 2006.

16. Diego Calvanese, Giuseppe De Giacomo, Domenico Lembo, Maurizio Lenzerini, and Riccardo Rosati. "Tractable Reasoning and Efficient Query Answering in Description Logics: The DL-Lite Family". In: JAR 39.3 (2007), pp. 385-429.

17. Diego Calvanese, Giuseppe De Giacomo, Domenico Lembo, Maurizio Lenzerini, and Riccardo Rosati. "EQLLite: Effective First-Order Query Processing in Description Logics". In: IJCAI-07. 2007, pp. 274-279.

18. Antonella Poggi, Domenico Lembo, Diego Calvanese, Giuseppe De Giacomo, Maurizio Lenzerini, and Riccardo Rosati. "Linking Data to Ontologies". In: JoDS X (2008), pp. 133-173.

19. Christian Bizer and Richard Cyganiak. D2R Server Publishing Relational Databases on the Semantic Web. (Poster Paper) 5th International Semantic Web Conference, Athens, USA. 2006.

20. Christian Bizer and Andy Seaborne. "D2RQ - Treating Non-RDF Databases as Virtual RDF Graphs". In: $3 r d$ International Semantic Web Conference (ISWC2004. 2004.

21. D2RQ website. Accessed October 2013. 2013. URL: http: //d2rq.org.

22. Andreas Langegger and Wolfram Wöß. "XLWrap - Querying and Integrating Arbitrary Spreadsheets with SPARQL". In: Proc. of ISWC2009. Springer, 2009, pp. 359-374. 
23. Nikos Bikakis, Nektarios Gioldasis, Chrisa Tsinaraki, and Stavros Christodoulakis. "Semantic Based Access over XML Data”. In: WSKS (1). Ed. by Miltiadis D. Lytras, Ernesto Damiani, John M. Carroll, Robert D. Tennyson, David E. Avison, Ambjörn Naeve, Adrian Dale, Paul Lefrere, Felix Tan, Janice C. Sipior, and Gottfried Vossen. Vol. 5736. Lecture Notes in Computer Science. Springer, 2009, pp. 259-267. ISBN: 978-3-642-04753-4.

24. Spyder. Online; Accessed April 2014. 2014. URL: http: //www.revelytix.com/content/spyder.

25. Diego Calvanese, Giuseppe De Giacomo, Domenico Lembo, Maurizio Lenzerini, Antonella Poggi, Mariano Rodriguez-Muro, Riccardo Rosati, Marco Ruzzi, and Domenico Fabio Savo. "The MASTRO system for ontology-based data access". In: Semantic Web 2.1 (2011), pp. 43-53.

26. Ontop website. Accessed October 2013. 2013. URL: http: //ontop.inf.unibz.it.

27. Yuan An, Alexander Borgida, and John Mylopoulos. "Inferring Complex Semantic Mappings Between Relational Tables and Ontologies from Simple Correspondences". In: OTM. 2005, pp. 1152-1169.

28. Eduard C. Dragut and Ramon Lawrence. "Composing Mappings Between Schemas Using a Reference Ontology”. In: OTM. 2004, pp. 783-800.

29. Wei Hu and Yuzhong Qu. "Discovering Simple Mappings Between Relational Database Schemas and Ontologies". In: ISWC/ASWC. 2007, pp. 225-238.

30. P. Papapanagiotou, P. Katsiouli, V. Tsetsos, C. Anagnostopoulos, and S. Hadjiefthymiades. "RONTO: relational to ontology schema matching". In: Semantic Web and Information Systems. AIS SIGSEMIS Bulletin 3 (2006).

31. Apache Jena website. Accessed October 2013. 2013. URL: http://jena. apache.org.

32. Stardog website. Accessed October 2013. 2013. URL: http: //stardog.com.

33. Tim Berners-Lee. Relational Databases on the Semantic Web. Online; Accessed April 2014. 1998. URL: http: // www . w3.org/DesignIssues/RDB-RDF.html.

34. Dan Connolly and Rob Crowell. dbview.py. Online; Accessed April 2014. 2002. URL: http : //dig . csail . mit . edu/2006/dbview/dbview . py.

35. Ljiljana Stojanovic, Nenad Stojanovic, and Raphael Volz. "Migrating data-intensive web sites into the Semantic Web". In: SAC. ACM, 2002, pp. 1100-1107.

36. Juan F. Sequeda, Syed Hamid Tirmizi, Oscar Corcho, and Daniel P. Miranker. "Survey of directly mapping SQL databases to the Semantic Web". In: The Knowledge Engineering Review 26.4 (2011), pp. 445-486.

37. Souripriya Das, Seema Sundara, and Richard Cyganiak, eds. R2RML: RDB to RDF Mapping Language. W3C Recommendation. Sept. 2012. URL: http: //www . w3 .org/TR/ 2012/REC-r2rml-20120927/.
38. Farid Cerbah. "Learning Highly Structured Semantic Repositories from Relational Databases: The RDBToOnto Tool". In: In Proc. of ESWC 2008. 2008.

39. OpenLink Virtuoso Universal Server. OpenLink Software. Online; Accessed June 2014. 2014. URL: http: //virtuoso. openlinksw.com/.

40. Basic Formal Ontology website. Ontology, Accessed October 2013. URL: http://www . ifomis . org/bfo/owl.

41. MySQL website. Accessed May 2014. 2013. URL: http: //www.mysql.com.

42. Marcelo Arenas, Alexandre Bertails, Eric Prud'hommeaux, and Juan Sequeda, eds. A Direct Mapping of Relational Data to RDF. W3C Recommendation. Sept. 2012. URL: http://www . w3. org/TR/rdb-direct-mapping/.

43. FactPages - Norwegian Petroleum Directorate. 2013. URL: http://factpages.npd.no/factpages/.

44. Tim Berners-Lee. Linked Data. 2009. URL: http : //www . w3. org/DesignIssues/LinkedData.html.

45. Martin G. Skjæveland and Espen H. Lian. "Benefits of Publishing the Norwegian Petroleum Directorate's FactPages as Linked Open Data". In: Norsk informatikkonferanse (NIK 2013). Tapir, 2013.

46. NPD FactMaps. 2013. URL: http : //npdmap1 .npd . no / website/NPDGIS/viewer.htm.

47. Norwegian Petroleum Directorate. DISKOS. Online; Accessed April 2013. 2009. URL: http: / /www.npd . no/en/ About-us/Collaboration-projects/DISKOS/.

48. Felix M. Gradstein, Erik Anthonissen, Harald Brunstad, Mike Charnock, Oyvind Hammer, Terje Hellem, and Kjell Sigve Lervik. "Norwegian Offshore Stratigraphic Lexicon (NORLEX)". In: Newsletters on Stratigraphy 44.1 (Oct. 2010), pp. 73-86.

49. International Commission on Stratigraphy. Stratigraphic Guide. Online; Accessed October 2013. 2013. URL: http : / / www . stratigraphy . org / index . php / ics stratigraphicguide.

50. OpenRefine. Accessed October 2013. URL: https : / / github.com/OpenRefine.

51. Asunción Gómez-Pérez, Mariano Fernández-López, and Oscar Corcho. Ontological Engineering: With Examples from the Areas of Knowledge Management, e-Commerce and the Semantic Web. (Advanced Information and Knowledge Processing). Secaucus, NJ, USA: Springer-Verlag New York, Inc., 2007. ISBN: 1846283965.

52. Cool URIs for the Semantic Web. W3C Interest Group Note. W3C, 2008. URL: http : / / www . w3 . org/TR/2008/NOTEcooluris-20081203/.

53. Tim Berners-Lee. Accessed October 2013. 2008. URL: http://www.w3.org/Provider/Style/URI.html.

54. Matthew Perry and John Herring, eds. OGC GeoSPARQL - A Geographic Query Language for RDF Data. OGC Implementation Standard. Sept. 2012. URL: http: //www . opengis.net/doc/IS/geosparql/1.0. 
55. Simon J D COX. International Chronostratigraphic Chart (2012). Ontology, Accessed October 2013. URL: http:// resource.geosciml .org/classifierscheme/ics/2012/ ischart.

56. Héctor Pérez-Urbina, Edgar Rodrıguez-Diaz, Michael Grove, George Konstantinidis, and Evren Sirin. "Evaluation of query rewriting approaches for OWL 2". In: Proc. of the Joint Workshop on Scalable and High-Performance Semantic Web Systems (SSWS+ HPCSW 2012) 943 (2012).

57. Christian Bizer and Andy Seaborne. "D2RQ-treating nonRDF databases as virtual RDF graphs". In: Proceedings of the 3rd International Semantic Web Conference (ISWC2004) (2004), p. 26.

58. Mariano Rodriguez-Muro and Diego Calvanese. "Quest, an OWL 2 QL Reasoner for Ontology-based Data Access". In: $O W L E D$. Ed. by Pavel Klinov and Matthew Horridge. Vol. 849. CEUR Workshop Proceedings. CEUR-WS.org, 2012.

59. Mariano Rodriguez-Muro, Josef Hardi, and Diego Calvanese. "Quest: Efficient SPARQL-to-SQL for RDF and OWL". In: International Semantic Web Conference (Posters $\mathcal{E}$ Demos). Ed. by Birte Glimm and David Huynh. Vol. 914. CEUR Workshop Proceedings. CEUR-WS.org, 2012.

60. R Development Core Team. R: A Language and Environment for Statistical Computing. ISBN 3-900051-07-0. R Foundation for Statistical Computing. Vienna, Austria, 2011. URL: http://www.R-project.org/.

61. Duncan Temple Lang. RCurl: General network (HTTP/FTP/...) client interface for $R$. R package version 1.95-4.1. 2013. URL: http : / / CRAN . R-project . org / package $=$ RCurl.

62. $c U R L$. curl and libcurl website. URL: http://curl . haxx . $\mathrm{se} /$.

63. Martin G. Skjæveland, Espen H. Lian, and Ian Horrocks. "Publishing the Norwegian Petroleum Directorate's FactPages as Semantic Web Data". In: The Semantic WebISWC 2013. Ed. by H. Alani, L. Kagal, A. Fokue, P. Groth, C. Biemann, J.X. Parreira, L. Aroyo, N. Noy, C. Welty, and K. Janowicz. Vol. 8219. LNCS. 2013.

64. Carsten Lutz, Dirk Walther, and Frank Wolter. "Conservative Extensions in Expressive Description Logics". In: Proc. of International Joint Conference on Artificial Intelligence (IJCAI-07). AAAI Press, 2007, pp. 453-459.

65. SPARQL 1.1 Query Language. W3C Recommendation. W3C, Mar. 2013. URL: http://www.w3.org/TR/2013/RECsparql11-query-20130321/.

66. Clark \& Parsia LLC. Stardog: Documentation: OWL 2. Online; Accessed September 2013. 2013. URL: http : // www. stardog.com/docs/ow12/.

67. Birte Glimm and Chimezie Ogbuji. SPARQL 1.1 Entailment Regimes. W3C Recommendation. W3C, 2013. URL: http : / / www . w3 . org / TR / 2013 / REC - sparql11 entailment-20130321/.
68. Ahmet Soylu, Martin Giese, Ernesto Jiménez-Ruiz, Evgeny Kharlamov, Dmitriy Zheleznyakov, and Ian Horrocks. "OptiqueVQS: towards an ontology-based visual query system for big data”. In: MEDES. Ed. by Latif Ladid, Antonio Montes, Peter A. Bruck, Fernando Ferri, and Richard Chbeir. ACM, 2013, pp. 119-126. ISBN: 978-14503-2004-7.

69. Martin Giese, Diego Calvanese, Peter Haase, Ian Horrocks, Yannis Ioannidis, Herald Kllapi, Manolis Koubarakis, Maurizio Lenzerini, Ralf Möller, Mariano Rodriguez-Muro, Özgür Özçep, Riccardo Rosati, Rudolf Schlatte, Michael Schmidt, Ahmet Soylu, and Arild Waaler. "Scalable EndUser Access to Big Data”. In: Big Data Computing. Ed. by Rajendra Akerkar. CRC Press, 2013.

70. Martin Giese, Ernesto Jiménez-Ruiz, Davide Lanti, Özgür Özçep, Martin Rezk, Riccardo Rosati, Ahmet Soylu, Guillermo Vega-Gorgojo, Arild Waaler, and Guohui Xiao. "Optique - Zooming in on Big Data". In: IEEE Computer 48.3 (2015), pp. 60-67.

71. M. Duerst and M. Suignard. Internationalized Resource Identifiers (IRIs). Tech. rep. The Internet Engineering Task Force, 2005. URL: http: //www . ietf . org/rfc/rfc3987. txt.

72. Graham Klyne and Jeremy J. Carroll. Resource Description Framework (RDF): Concepts and Abstract Syntax. W3C Recommendation. W3C, Feb. 2004. URL: http: // Www. w3.org/TR/rdf-concepts/. 